\title{
Polaronic effects in a Gaussian quantum dot
}

\author{
Sonay Yanar ${ }^{\mathrm{a}}$, Ata Sevim ${ }^{\mathrm{a}}$, B. Boyacioglu ${ }^{\mathrm{b}}$, Mesude Saglam ${ }^{\mathrm{a}}$, \\ Soma Mukhopadhyaya ${ }^{\mathrm{c}, 1}$, Ashok Chatterjee ${ }^{\mathrm{c}, *, 2}$ \\ a Department of Physics, Faculty of Sciences, Ankara University, 06100 Tandogan-Ankara, Turkey \\ ${ }^{\mathrm{b}}$ Vocational School of Health, Ankara University, 06290 Kecioren, Ankara, Turkey \\ ${ }^{\mathrm{c}}$ Department of Physics, Bilkent University, 06800 Bilkent, Ankara, Turkey
}

Received 13 August 2007; received in revised form 13 November 2007; accepted 14 November 2007

Available online 21 February 2008

\begin{abstract}
The problem of an electron interacting with longitudinal-optical (LO) phonons is investigated in an $N$-dimensional quantum dot with symmetric Gaussian confinement in all directions using the Rayleigh-Schrödinger perturbation theory, a variant of the canonical transformation method of Lee-Low-Pines, and the sophisticated apparatus of the Feynman-Haken path-integral technique for the entire range of the coupling parameters and the results for $N=2$ and $N=3$ are obtained as special cases. It is shown that the polaronic effects are quite significant for small dots with deep confining potential well and the parabolic potential is only a poor approximation of the Gaussian confinement. The Feynman-Haken path-integral technique in general gives a good upper bound to the ground state energy for all values of the system parameters and therefore is used as a benchmark for comparison between different methods. It is shown that the perturbation theory yields for the ground state polaron self-energy a simple closed-form analytic expression containing only Gamma functions and in the weak-coupling regime it provides the lowest energy because of an efficient partitioning of the Gaussian potential and the subsequent use of a mean-field kind of treatment. The polarization potential, the polaron radius and the number of virtual phonons in the polaron cloud are obtained using the Lee-Low-Pines-Huybrechts method and their variations with respect to different parameters of the system are discussed.
\end{abstract}

(C) 2007 Elsevier Ltd. All rights reserved.

Keywords: Quantum dot; Gaussian confinement; Polaronic effect

\footnotetext{
* Corresponding author. Tel.: +90 312290 2673; Fax: +90 3122664779.

E-mail address: ashok@fen.bilkent.edu.tr (A. Chatterjee).

1 On leave from Shadan Institute of P.G. Studies, Khairatabad, Hyderabad, India.

2 On leave from School of Physics, University of Hyderabad, Hyderabad, India.
} 


\section{Introduction}

The last two decades have witnessed a hectic pace of activity with a flurry of investigations in the area of low-dimensional systems and consequently a huge amount of literature has now piled up in this field with a rich variety of physics both at the fundamental level unravelling some of the basic tenets of quantum mechanics and also at the applied level heralding the ushering of a new era in micro-electronic technology [1]. In this context, polar semiconductor quantum dot structures have been, in particular, the focus of attention for their potential applications in nano-electronic and optoelectronic devices like single electron transistors, quantum dot lasers, ultra-fast computers and so on. Since the natural length scale in a quantum dot is of the order of a few nanometers where one would expect pronounced quantum effects like charge quantization and Coulomb blockade, a quantum dot can be considered as a tiny laboratory to test the veracity of the predictions of quantum mechanics and thus has an intrinsic appeal from the point of view of fundamental physics. On the other hand, quantum dot structures can be realized in different shapes and sizes because of the recent advent in modern micro-fabrication techniques like molecular beam epitaxy and self-assembling and that provides a tremendous design flexibility for their use in semiconductor technology. Another important feature that makes the study of quantum dots so exciting is that these structures involve several length and energy scales which are of the same order of magnitude and therefore their interplay gives rise to a host of novel and complex physical phenomena which are extremely different from those of their bulk counterparts. Because of the nano-scale extensions in all the directions of space, the quantum dots possess discrete energy levels that can be tuned at will and furthermore, the number of electrons in a quantum dot can also be varied at will by manipulating the confining potential or the so-called gate voltage and thus one can obtain an energy spectra of the desired properties. The reduced dimensionality with enormous design flexibility, the possibility of achieving a finite but variable particle number system, the presence of $\delta$-function-like density of states and several competing length and energy scales have indeed made this new research area of low-dimensional systems extremely fascinating with lots of challenges and also opportunities and opened up a new frontier in condensed matter physics and materials science with tremendous potentiality to revolutionize modern optoelectronic technology.

The theoretical investigation of a quantum dot requires the knowledge of the attractive confining potential. One of the ideal methods to obtain such a potential would have been to perform scattering experiments and then use the scattering data to obtain the potential parameters using the inverse scattering method. However, to our knowledge, such investigations are at the moment lacking. In the simplest approach, one can then assume the electron to be confined in an infinite potential well to simulate the actual confining potential. This model is however not very realistic since the constant potential inside the dot implies that the motion of electrons is completely free in a quantum dot which is of course far from reality. Sikorski and Merkt [2] have observed experimentally that the resonance frequencies of quantum dots are independent of the number of electrons in the dot which indicates that the electron-electron interaction has no effect on the excitation spectrum of a quantum dot. Later, Meurer et al. [3] obtained the energy spectrum of a few-electron quantum dot using the far-infrared spectroscopy on GaAs quantum dot and showed that the electron number has at best a very feeble influence on the resonance frequencies. Independence of the dipole transitions on the electron number was also approximately observed in GaAs-GaAlAs quantum dots by Ashoori et al. [4] and in InGaAs-GaAs-AlAs system by Drexler et al. [5]. All these results suggest an interesting feature about the quantum dot namely, the validity of the Kohn theorem or more precisely the generalization of it in a quantum dot. 
Kohn's theorem [6] states that the cyclotron frequency of a translationally invariant electron system is independent of the electron density and of the form of the electron-electron interaction. This theorem is a consequence of the fact that the electric dipole of the radiation couples only to the centre of mass of the electrons and does not affect the relative motion. Peeters [7] has demonstrated that the position of the resonance lines in the magneto-optical absorption of a quantum dot with a parabolic confinement potential is also independent of the electron-electron interaction and the number of electrons in a quantum dot. Similar results have also been obtained by Yip [8] and Li et al. [9]. These results thus indicate the validity of the so-called generalized Kohn's theorem in a quantum dot according to which one can conclude that the bare confining potentials are almost parabolic in these systems. Consequently, a large number of theoretical investigations have been carried out in this area in the last two decades taking the confinement potential as parabolic [10].

However, in recent years a few groups [11] have shown that their experimental results deviate from what would be expected from the generalized Kohn theorem. Their observations reveal that the confining potential should be non-parabolic i.e., anharmonic and should have the shape of a finite potential well. This has provided, understandably, a renewed impetus on the research of the electronic energy spectra and the associated phenomena in quantum dots. Using a 3D spherical rectangular potential well of finite depth, Szafran et al. [12] and Bednarek et al. [13] have been able to successfully describe, albeit qualitatively, the charging of quantum dots. Subsequently, Szafran et al. [14] have quantitatively explained the capacitance-spectroscopy data for selfassembled quantum dots simulating the confining potential by a 3D cylindrical potential well. Recently, Adamowsky et al. [15] have proposed a Gaussian attractive confining potential for the investigation of the properties of excess electrons in quantum dots. This potential has a finite depth and in the neighbourhood of the dot centre would behave like a parabolic potential and would also approximately satisfy the generalized Kohn theorem. Furthermore, in contrast to the rectangular potential well, it is continuous at the dot boundaries and this makes it easier to be handled mathematically. Also it has a central minimum as required for a physical potential and the force experienced by the particles within this potential well is non-zero which is also a desirable feature. The other advantages with the Gaussian confining potential vis-a-vis a parabolic potential are that the former allows for, in addition to the excitations, also the ionization and tunnelling processes. Of course, one can use power law anharmonic potentials, but these potentials suffer from divergence syndrome at large distances, while the Gaussian potential is by construction bound to give convergent results. Of course one may argue that in a quantum dot the spatial coordinates never extend to a very large value to lead to any divergence problem, nevertheless, it is always appealing to work with a prescription that is mathematically sound and works in all limits. We would like to mention in passing that the Gaussian potential has proved to be a useful potential in various branches of physics and has been solved approximately for a single particle problem by several authors [16].

Since most of the quantum dot structures available today are made of polar semiconductors, and the electron-phonon interaction energy scale is almost comparable to the other energy scales of the problem, one expects that the electron-longitudinal-optical (LO) phonon interaction will have pronounced effects on the electronic states of a polar quantum dot leading to the formation of polarons and furthermore these polaronic effects should be size dependent and therefore tunable. Though, a large number of investigations have been carried out in the last two decades to study the polaronic effects in parabolic quantum dots and in quantum dots with square and spherical well confining potentials, there has not been any investigation, to our knowledge, with a Gaussian confinement. Since several authors have recently shown quite unequivocally that the 
parabolic potential is only a poor approximation of the more realistic Gaussian potential, it would be interesting to study the polaronic effects in a quantum dot with a Gaussian confinement. The purpose of the present study is to make an attempt in this direction.

\section{The model}

The hamiltonian for an electron of Bloch mass $m$ moving in an $N$-dimensional (ND) Gaussian potential $V^{\prime}$ and interacting with the LO phonons of dispersionless frequency $\omega_{o}$ of the system may be written by modifying the Fröhlich hamiltonian [17,18], as

$$
H^{\prime}=\frac{\vec{p}^{\prime 2}}{2 m}+V^{\prime}\left(\vec{r}^{\prime}\right)+\hbar \omega_{o} \sum_{\vec{q}^{\prime}} b_{\vec{q}^{\prime}}^{\dagger} b_{\vec{q}^{\prime}}+\sum_{\vec{q}^{\prime}}\left(\xi_{\vec{q}^{\prime}}^{\prime} \mathrm{e}^{-i \vec{q}^{\prime} \cdot \vec{r}^{\prime}} b_{\vec{q}^{\prime}}^{\dagger}+h . c .\right),
$$

where all vectors are $N$-dimensional. Here $\vec{r}^{\prime}$ refers to the $N$-dimensional position vector of the electron, $\vec{p}^{\prime}$ is the corresponding linear momentum operator, $b_{\vec{q}^{\prime}}^{*}\left(b_{\vec{q}^{\prime}}\right)$ is the creation (annihilation) operator for an LO phonon of wave vector $\vec{q}^{\prime}$ with frequency $\omega_{o}$ and $\xi_{\vec{q}^{\prime}}$ is the electron-phonon interaction coefficient and $V^{\prime}\left(\vec{r}^{\prime}\right)$ is the confining potential which we take as

$$
V^{\prime}\left(\vec{r}^{\prime}\right)=-V_{o}^{\prime} \mathrm{e}^{-r^{\prime 2} / 2 R^{\prime 2}} .
$$

We shall work in the Feynman units [19] in which the energy is scaled by $\hbar \omega_{o}$, length by the weak-coupling polaron radius, $r_{o}=\left(\hbar / m \omega_{o}\right)^{1 / 2}$ and the wave vector by $q_{o}=1 / r_{o}$. This is equivalent to putting $\hbar=m=\omega_{o}=1$. In these units the dimensionless Hamiltonian reads

$$
H=\frac{\vec{p}^{2}}{2}-V_{o} \mathrm{e}^{-r^{2} / 2 R^{2}}+\sum_{\vec{q}} b_{\vec{q}}^{\dagger} b_{\vec{q}}+\sum_{\vec{q}}\left(\xi_{\vec{q}} \mathrm{e}^{-i \vec{q} \cdot \vec{r}} b_{\vec{q}}^{\dagger}+h . c .\right),
$$

where everything is dimensionless; $H=H^{\prime} / \hbar \omega_{o}, \vec{r}=\vec{r}^{\prime} / r_{0}, \vec{q}=\vec{q}^{\prime} / q_{o}, \vec{p}=\vec{p}^{\prime} / \hbar q_{o}$, $V_{o}=V_{o}^{\prime} / \hbar \omega_{o}, R=R^{\prime} / r_{o}$, and $\xi_{\vec{q}}=\xi_{\vec{q}^{\prime}}^{\prime} / \hbar \omega_{o}$ is given by [20],

$$
\left|\xi_{\vec{q}}\right|^{2}=\left[\frac{\Gamma\left(\frac{(N-1)}{2}\right) 2^{(N-3 / 2)} \pi^{(N-1) / 2}}{v_{N} q^{N-1}}\right] \alpha,
$$

where $N$ is the dimensionality of the system under consideration, $v_{N}$ is the dimensionless volume of the $N$-dimensional crystal, $V_{o}$ is the depth of the confining potential in dimensionless unit and $R$ is the range of the potential giving some kind of a measure of the effective confinement length or the size of the quantum dot and $\alpha$ is the dimensionless electron-phonon coupling constant. In two and three dimensions we get the usual expressions:

$$
\left|\xi_{\vec{q}}\right|^{2}=\left(\frac{\sqrt{2} \pi}{v q}\right) \alpha, \quad \text { for } 2 D
$$

and

$$
\left|\xi_{\vec{q}}\right|^{2}=\left(\frac{2 \sqrt{2} \pi}{v q^{2}}\right) \alpha, \text { for } 3 D,
$$

where $v$ represents the volume in the corresponding dimension. 


\section{Formulation}

\subsection{Rayleigh-Schrödinger perturbation theory (RSPT)}

We assume that the deviation of the confining potential from the parabolic potential is small enough so that one can treat it as a parabolic potential plus a perturbation. This is a reasonable assumption for small $r$ and since in a quantum dot $r$ will be generally small, it can be considered as a fairly good approximation. So we rewrite the hamiltonian (3) as

$$
\begin{aligned}
H= & \frac{\vec{p}^{2}}{2}+\frac{1}{2} \tilde{\omega}_{h}^{2} r^{2}-V_{o}+\sum_{\vec{q}} b_{\vec{q}}^{\dagger} b_{\vec{q}}-\lambda\left[\frac{1}{2} \tilde{\omega}_{h}^{2} r^{2}+V_{o}\left(\mathrm{e}^{-r^{2} / 2 R^{2}}-1\right)\right] \\
& +\sum_{\vec{q}}\left(\xi_{\vec{q}} \mathrm{e}^{-i \vec{q} \cdot \vec{r}} b_{\vec{q}}^{\dagger}+\text { h.c. }\right),
\end{aligned}
$$

where $\tilde{\omega}_{h}^{2}=V_{o} / R^{2}$, and $\lambda=0$ for a parabolic quantum dot (PQD) and $\lambda=1$ for a Gaussian quantum dot (GQD). The unperturbed problem satisfies the Schrödinger equation

$$
\begin{aligned}
H_{o}\left[\Phi_{[j]}(\vec{r}) \Psi_{p h}\right] & =E_{[j],[n]}^{0}\left[\Phi_{[j]}(\vec{r}) \Psi_{p h}\right] \\
& =\left(\frac{\vec{p}^{2}}{2}+\frac{1}{2} \tilde{\omega}_{h}^{2} r^{2}-V_{o}+\sum_{\vec{q}} b_{\vec{q}}^{\dagger} b_{\vec{q}}\right)\left[\Phi_{[j]}(\vec{r}) \Psi_{p h}\right] \\
& =\left(E_{[j]}+\sum_{\vec{q}} n_{\vec{q}}\right)\left[\Phi_{[j]}(\vec{r}) \Psi_{p h}\right],
\end{aligned}
$$

where $\Phi_{[j]}(\vec{r})$ is the unperturbed electronic wave function given by

$$
\begin{aligned}
\Phi_{[j]}(\vec{r})= & \Phi_{j_{1}, j_{2}, j_{3} \ldots j_{N}}(\vec{r})=\left[\frac{\tilde{\omega}_{h}^{N / 2}}{\pi^{N / 2} 2^{j_{1}+j_{2}+\cdots+j_{N}} j_{1} ! j_{2} ! j_{3} ! \ldots j_{N} !}\right]^{1 / 2} \\
& \times H_{j_{1}}\left(\sqrt{\tilde{\omega}_{h}} x_{1}\right) H_{j_{2}}\left(\sqrt{\tilde{\omega}_{h}} x_{2}\right) \ldots H_{j_{N}}\left(\sqrt{\tilde{\omega}_{h}} x_{N}\right) \times \mathrm{e}^{-\frac{1}{2} \tilde{\omega}_{h} r^{2}},
\end{aligned}
$$

$E_{[n]}$ is the corresponding electronic energy which assumes the following expression

$$
E_{[j]}=E_{j_{1}, j_{2}, j_{3} \ldots j_{N}}=\left(j_{1}+j_{2}+j_{3}+\cdots+j_{N}+\frac{N}{2}\right) \tilde{\omega}_{h}-V_{o},
$$

and $\Psi_{p h}$ is the eigenfunction of the free phonon hamiltonian, $\sum_{\vec{q}} b_{\vec{q}}^{\dagger} b_{\vec{q}}$ which can be written as

$$
\left|\Psi_{p h}\right\rangle \equiv|[n]\rangle=\prod_{\vec{q}}\left|n_{\vec{q}}\right\rangle
$$

with the corresponding energy given by

$$
\epsilon_{p h}=\sum_{\vec{q}} n_{\vec{q}}
$$

where $n_{\vec{q}}$ is the number of phonons in the state $\vec{q}$. 
We are presently interested in the ground state (GS) of the system for which the unperturbed wave functions and the energy reduce to

$$
\begin{aligned}
& \Phi_{o}(\vec{r})=\left[\frac{\tilde{\omega}_{h}^{N / 2}}{\pi^{N / 2}}\right]^{1 / 2} \mathrm{e}^{-\frac{1}{2} \tilde{\omega}_{h} r^{2}}, \\
& \left|\Psi_{p h}^{0}\right\rangle=|[0]\rangle=\prod_{\vec{q}}\left|0_{\vec{q}}\right\rangle,
\end{aligned}
$$

with

$$
b_{\vec{q}}\left|0_{\vec{q}}\right\rangle=0, \quad \forall \vec{q},
$$

and

$$
E_{o}=\left(\frac{N}{2}\right) \tilde{\omega}_{h}-V_{o}
$$

and

$$
\epsilon_{p h}^{0}=0
$$

The perturbation to the exactly soluble unperturbed hamiltonian $H_{O}$ consists of two terms:

$$
H_{\text {per }}=H_{1}+H_{2},
$$

where

$$
H_{1}=-\lambda\left[\frac{1}{2} \tilde{\omega}_{h}^{2} r^{2}+V_{o}\left(\mathrm{e}^{-r^{2} / 2 R^{2}}-1\right)\right],
$$

and

$$
H_{2}=\sum_{\vec{q}}\left(\xi_{\vec{q}} \mathrm{e}^{-i \vec{q} \cdot \vec{r}} b_{\vec{q}}^{\dagger}+\text { h.c. }\right) .
$$

Since $H_{1}$ cannot be very large, we shall obtain its contribution by the first-order perturbation theory at the mean-field level or in other words we shall assume that the sole effect of $H_{1}$ is to renormalize the frequency of the unperturbed parabolic potential according to the first-order perturbation theory. Therefore we rewrite $H_{1}$ as

$$
\begin{aligned}
H_{1} & =-\lambda\left[\frac{1}{2} \tilde{\omega}_{h}^{2}+\left(\frac{V_{o}}{r^{2}}\right)\left(\mathrm{e}^{-r^{2} / 2 R^{2}}-1\right)\right] r^{2} \\
& \approx-\lambda\left\langle\Phi_{o}(\vec{r})\left|\left[\frac{1}{2} \tilde{\omega}_{h}^{2}+\frac{V_{o}}{r^{2}}\left(\mathrm{e}^{-\frac{r^{2}}{2 R^{2}}}-1\right)\right]\right| \Phi_{o}(\vec{r})\right\rangle r^{2} \\
& =\lambda V_{o}\left[\frac{2 \tilde{\omega}_{h}}{N-2}-\frac{1}{R^{2}}-\frac{2 \tilde{\omega}_{h}^{\frac{N}{2}}}{(N-2)\left(\tilde{\omega}_{h}+\frac{1}{2 R^{2}}\right)^{\frac{N}{2}-1}}\right] r^{2} .
\end{aligned}
$$


The Gaussian quantum dot problem now essentially reduces to an effective parabolic quantum dot problem described by

$$
\tilde{H}=H_{o}^{\mathrm{eff}}+H_{2}=\frac{\vec{p}^{2}}{2}+\frac{1}{2} \omega_{h}^{2} r^{2}-V_{o}+\sum_{\vec{q}} b_{\vec{q}}^{\dagger} b_{\vec{q}}+H_{2},
$$

where

$$
\omega_{h}=\left[(1-\lambda) \tilde{\omega}_{h}^{2}+\frac{4 \lambda V_{0} \tilde{\omega}_{h}}{(N-2)}\left(1-\frac{1}{\left(1+\frac{\tilde{\omega}_{h}}{2 V_{0}}\right) \frac{N}{2}-1}\right)\right]^{1 / 2}
$$

The first-order perturbative correction to $H_{o}^{\text {eff }}$ due to $H_{2}$ is zero, for the electron-phonon interaction is linear in the phonon creation and annihilation operators, and so we shall treat this term by the second-order perturbation theory. The calculation of the second-order perturbation correction of course always poses a problem for it requires one to calculate a summation over infinite intermediate states. However, in the present problem, the unperturbed system being approximated as an $\mathrm{N}$-dimensional harmonic oscillator, the summation over infinite intermediate states essentially boils down to the calculation of the Green function of the harmonic oscillator in $N$-dimensions, which is fortunately an exactly soluble problem.

The second-order perturbative correction $\Delta E_{\mathrm{RSPT}}^{(2)}$ due to $H_{2}$ can be written as

$$
\Delta E_{\mathrm{RSPT}}^{(2)}=\sum_{[j],[n]} \frac{\left|\left\langle\Phi_{[j]}\left|\left\langle[n]\left|\sum_{\vec{q}}\left(\xi_{\vec{q}} \mathrm{e}^{-i \vec{q} \cdot \vec{r}} b_{\vec{q}}^{\dagger}\right)\right|[0]\right\rangle\right| \Phi_{o}\right\rangle\right|^{2}}{\left(E_{[0],[0]}-E_{[j],[n]}\right)},
$$

where $\Phi_{[j]}(\vec{r})$ and $E_{[j]}$ are given by (9) and (10) with $\tilde{\omega}_{h}$ replaced by $\omega_{h}$. The summation over the phonon states can be performed easily and we get

$$
\Delta E_{\mathrm{RSPT}}^{(2)}=-\sum_{[j], \vec{q}} \frac{\left|\left\langle\Phi_{[j]}\left|\xi_{\vec{q}} \mathrm{e}^{-i \vec{q} \cdot \vec{r}}\right| \Phi_{o}\right\rangle\right|^{2}}{E_{[j]}-E_{[0]}+1} .
$$

Using the identity,

$$
\frac{1}{E_{m}-E_{0}+1}=\int_{0}^{\infty} \mathrm{e}^{-\left(E_{m}-E_{0}+1\right) t} \mathrm{~d} t
$$

the $N$-dimensional inverse Coulomb Fourier transform

$$
\sum_{\vec{q}} \frac{\mathrm{e}^{-i \vec{q} \cdot\left(\vec{r}-\vec{r}^{\prime}\right)}}{\vec{q}^{N-1}}=\left[\frac{v_{N}}{2^{N-1} \pi^{(N-1) / 2} \Gamma\left(\frac{N-1}{2}\right)}\right] \frac{1}{\left|\vec{r}-\vec{r}^{\prime}\right|},
$$

and the Slater sum rule (which is related to the oscillator Green function)

$$
\begin{aligned}
& \sum_{n} \frac{1}{2^{n} n !} H_{n}(\sqrt{\lambda} x) H_{n}\left(\sqrt{\lambda} x^{\prime}\right) \mathrm{e}^{-\left[\frac{\lambda}{2}\left(x^{2}+x^{\prime 2}\right)+2 n p\right]} \\
& =\frac{\mathrm{e}^{p}}{\sqrt{2 \sinh (2 p)}} \mathrm{e}^{-\frac{\lambda}{4}\left[\left(x+x^{\prime}\right)^{2} \tanh p+\left(x-x^{\prime}\right)^{2} \operatorname{coth} p\right]},
\end{aligned}
$$


for all the $N$-set of coordinates $\vec{r}$ and $\vec{r}^{\prime}$, we can transform Eq. (25) to

$$
\begin{aligned}
\Delta E_{\mathrm{RSPT}}^{(2)}= & -\frac{\alpha}{\sqrt{2}}\left(\frac{\omega_{h}}{\pi}\right)^{N} \int_{0}^{\infty} \mathrm{d} t \frac{\mathrm{e}^{\left(\frac{N}{2} \omega_{h}-1\right) t}}{\left[2 \sinh \left(\omega_{h} t\right)\right]^{N / 2}} \times \iint \mathrm{d} \vec{r} \mathrm{~d} \vec{r}^{\prime} \frac{1}{\left|\vec{r}-\vec{r}^{\prime}\right|} \\
& \times \mathrm{e}^{-\left(\frac{\omega_{h}}{4}\right)\left[\left(1+\tanh \left(\frac{\omega_{h} t}{2}\right)\right)\left(\vec{r}+\vec{r}^{\prime}\right)^{2}\right]} \times \mathrm{e}^{-\left(\frac{\omega_{h}}{4}\right)\left[\left(1+\operatorname{coth}\left(\frac{\omega_{h} t}{2}\right)\right)\left(\vec{r}-\vec{r}^{\prime}\right)^{2}\right]} .
\end{aligned}
$$

Using the transformations

$$
\frac{\vec{r}+\vec{r}^{\prime}}{\sqrt{2}}=\vec{u} ; \quad \frac{\vec{r}-\vec{r}^{\prime}}{\sqrt{2}}=\vec{v},
$$

the integrations over the spatial coordinates can be performed easily and the t-integration can be simplified by substituting $t=-[\ln x] / \omega_{h}$ to finally obtain for the total energy

$$
E_{\mathrm{RSPT}}^{\mathrm{ND}}=\frac{N}{2} \omega_{h}-V_{0}-\left(\frac{\alpha \sqrt{\pi}}{2 \sqrt{\omega_{h}}}\right)\left[\frac{\Gamma\left(\frac{N-1}{2}\right)}{\Gamma\left(\frac{N}{2}\right)}\right]\left[\frac{\Gamma\left(\frac{1}{\omega_{h}}\right)}{\Gamma\left(\frac{1}{\omega_{h}}+\frac{1}{2}\right)}\right] .
$$

It is worthwhile to note that we have obtained the total energy of the system analytically in the form of a closed-form expression involving only the gamma functions. The polaronic correction to the total electron energy or in other words the polaron self-energy can now be obtained by subtracting from the expression Eq. (31), the energy for $\alpha=0$. Thus we obtain

$$
\begin{aligned}
\Delta E_{\mathrm{RSPT}}^{\mathrm{ND}} & =\left[E_{\mathrm{RSPT}}^{\mathrm{ND}}(\alpha)-E_{\mathrm{RSPT}}^{\mathrm{ND}}(\alpha=0)\right] \\
& =-\left(\frac{\alpha \sqrt{\pi}}{2 \sqrt{\omega_{h}}}\right)\left[\frac{\Gamma\left(\frac{N-1}{2}\right)}{\Gamma\left(\frac{N}{2}\right)}\right]\left[\frac{\Gamma\left(\frac{1}{\omega_{h}}\right)}{\Gamma\left(\frac{1}{\omega_{h}}+\frac{1}{2}\right)}\right] .
\end{aligned}
$$

If we define an effective length $l$ in analogy with a parabolic dot as

$$
l=\left[\omega_{h}\right]^{-1 / 2}
$$

then the polaronic correction can be written as

$$
\Delta E_{\mathrm{RSPT}}^{\mathrm{ND}}=-\left(\frac{\alpha \sqrt{\pi}}{2}\right)\left[\frac{\Gamma\left(\frac{N-1}{2}\right)}{\Gamma\left(\frac{N}{2}\right)}\right]\left[\frac{\Gamma\left(l^{2}+1\right)}{l \Gamma\left(l^{2}+\frac{1}{2}\right)}\right] .
$$

The polaron self-energy thus depends crucially on two parameters $\alpha$ and $l$. It is worthwhile to point out that according to the above definition of $l$, the confinement length is a very complicated function of the depth and range of the Gaussian potential. We shall therefore consider the range of the potential as an effective confinement length for the sake of simplicity. At this point it would be interesting to discuss some limiting cases.

(i) Weak-confinement (WC) limit

In this limit, $V_{o}$ is small and the range of the confining potential, $R$ is large so that both $\tilde{\omega}_{h}$ and $\omega_{h}$ tend to zero and consequently $l \rightarrow \infty$. We can use in this limit the asymptotic formula [21]

$$
\frac{\Gamma(z+1)}{\sqrt{z} \Gamma\left(z+\frac{1}{2}\right)} \rightarrow\left(1+\frac{1}{8 z}\right)
$$


so that the most dominant contribution from Eq. (34) becomes

$$
\Delta E_{\mathrm{RSPT}}^{\mathrm{ND}, \mathrm{WC}}=-\left(\frac{\alpha \sqrt{\pi}}{2}\right)\left[\frac{\Gamma\left(\frac{N-1}{2}\right)}{\Gamma\left(\frac{N}{2}\right)}\right] .
$$

In $2 \mathrm{D}$ and $3 \mathrm{D}$, one gets respectively

$$
\Delta E_{\mathrm{RSPT}}^{2 \mathrm{D}, \mathrm{WC}}=-\frac{\alpha \pi}{2},
$$

and

$$
\Delta E_{\mathrm{RSPT}}^{3 \mathrm{D}, \mathrm{WC}}=-\alpha,
$$

which are the well-known bulk polaron limits in the weak-coupling regime.

(ii) Strong-confinement (SC) limit

In the strong-confinement case, we assume $V_{0}$ to be large and $R$ to be small so that $l^{2}$ becomes small. However in this limit, the product $\sqrt{ } V_{0} R$ is assumed to be finite, preferably less than 1. We can then write

$$
\begin{aligned}
& \Gamma(1+z)=\Gamma(1)+z \Gamma^{\prime}(1)+\cdots \approx 1+z \Psi(1) \\
& \Gamma\left(\frac{1}{2}+z\right)=\Gamma\left(\frac{1}{2}\right)+z \Gamma^{\prime} \frac{1}{2}+\cdots \approx \sqrt{\pi}+z \sqrt{\pi} \Psi\left(\frac{1}{2}\right),
\end{aligned}
$$

where

$$
\begin{aligned}
& \Psi(z)=\frac{\mathrm{d}}{\mathrm{d} z} \ln \Gamma(z)+\cdots \approx \sqrt{\pi}+z \sqrt{\pi} \Psi\left(\frac{1}{2}\right)=\frac{\Gamma^{\prime}(z)}{\Gamma(z)} \\
& \Psi(1)=-0.5772 \\
& \Psi\left(\frac{1}{2}\right)=\Psi(1)-2 \ln 2
\end{aligned}
$$

and therefore

$$
\frac{\Gamma(1+z)}{\Gamma\left(z+\frac{1}{2}\right)} \approx \frac{(1+2 z \ln 2)}{\sqrt{\pi}},
$$

so that the polaron self-energy reduces to

$$
\Delta E_{\mathrm{RSPT}}^{\mathrm{ND}, \mathrm{SC}}=-\left(\frac{\alpha}{2}\right)\left[\frac{\Gamma\left(\frac{N-1}{2}\right)}{\Gamma\left(\frac{N}{2}\right)}\right] \frac{1}{l} .
$$

In $2 \mathrm{D}$ and $3 \mathrm{D}$, one then obtains respectively

$$
\Delta E_{\mathrm{RSPT}}^{2 \mathrm{D}, \mathrm{SC}} \approx-\left(\frac{\alpha \sqrt{\pi}}{2}\right)\left(\frac{V_{0}}{\sqrt{ } R}\right)^{1 / 4},
$$

and

$$
\Delta E_{\mathrm{RSPT}}^{3 \mathrm{D}, \mathrm{SC}} \approx-\left(\frac{\alpha}{\sqrt{\pi}}\right)\left(\frac{V_{0}}{\sqrt{ } R}\right)^{1 / 2} .
$$


These are of course new results and therefore cannot be compared with any previous values. However, there should be a word of caution here. The above limiting results have to be used carefully because we have derived them using certain approximations like $l^{2}$ is small etc., and therefore one should be allowed to use them only when the right conditions are met, and that would depend on the relative values of $V_{0}$ and $R$. For the entire range of the confining potential, however, we have to obtain the results numerically. In Section 4 we shall discuss the numerical results.

One can however notice that the above formalism, albeit in general applicable to any dimensional system, falls through in $2 \mathrm{D}$. We would therefore like to develop another perturbation theory that may work in all dimensions. To that end, we expand the Gaussian potential as

$$
-V_{0} \mathrm{e}^{-r^{2} / 2 R^{2}}=-V_{0}+\frac{V_{0}}{2 R^{2}} r^{2}-\frac{V_{0}}{8 R^{4}} r^{4}+\frac{V_{0}}{48 R^{6}} r^{6}+\cdots,
$$

and approximate it as

$$
-V_{0} \mathrm{e}^{-r^{2} / 2 R^{2}}=-V_{0}+\frac{1}{2}\left[\tilde{\omega}_{h}^{2}-\frac{\tilde{\omega}_{h}^{2}}{4 R^{2}}\left\langle r^{2}\right\rangle+\frac{\tilde{\omega}_{h}^{2}}{24 R^{4}}\left\langle r^{4}\right\rangle+\cdots\right] r^{2},
$$

where the averaging is done with respect to the oscillator wave function corresponding to the frequency $\tilde{\omega}_{h}$. The hamiltonian (3) is thus reduced to

$$
\tilde{H}=H_{o}^{\text {eff }}+H_{2}=\frac{\vec{p}^{2}}{2}+\frac{1}{2} \omega_{h}^{2} r^{2}-V_{o}+\langle f(r)\rangle+\sum_{\vec{q}} b_{\vec{q}}^{\dagger} b_{\vec{q}}+H_{2},
$$

where $\omega_{h}$ is now given by

$$
\omega_{h}=\left[\tilde{\omega}_{h}^{2}-\frac{\lambda N \tilde{\omega}_{h}}{8 R^{2}}+\frac{\lambda N(N+2)}{96 R^{2}}\right]^{1 / 2},
$$

and $\langle f(r)\rangle$ contains contributions from the higher-order terms of the Gaussian potential which we have neglected assuming that for the polaronic correction it has negligible effect. The rest of the calculation is along the same lines as delineated above.

\subsection{The Feynman-Haken path-integral method (FHPIM)}

We are interested in the GS of the hamiltonian (3) and the relevant transformation function can be shown to be given by $[17,19]$,

$$
K_{00}\left(\vec{r}_{b}, t_{b} ; \vec{r}_{a}, t_{a}\right)=\int D \vec{r}(t) \mathrm{e}^{i S},
$$

where the index " 00 " refers to the transition from a zero-phonon state to a zero-phonon state, the integration is from all paths from $\vec{r}\left(t_{a}\right)$ to $\vec{r}\left(t_{b}\right)$ and $S$ is a non-local classical action given by

$$
S=\int_{t_{a}}^{t_{b}}\left(\frac{1}{2} \dot{\vec{r}}^{2}-V_{0} \mathrm{e}^{-r^{2} / 2 R^{2}}\right) \mathrm{d} t+\frac{1}{2} \sum_{\vec{q}} \iint \mathrm{d} t \mathrm{~d} s\left|\xi_{\vec{q}}\right|^{2} \mathrm{e}^{i \vec{q} \cdot(\vec{r}(t)-\vec{r}(s))} \mathrm{e}^{i|t-s|} .
$$

Performing the transforming $t \rightarrow i t$ and $s \rightarrow-i s$, we obtain

$$
K_{00}\left(\vec{r}_{b}, t_{b} ; \vec{r}_{a}, t_{a}\right)=\int_{a}^{b} D \vec{r}(t) \mathrm{e}^{S}
$$


where the action reads

$$
S=\int_{t_{a}}^{t_{b}}\left(-\frac{1}{2} \dot{\vec{r}}^{2}-V_{0} \mathrm{e}^{-r^{2} / 2 R^{2}}\right) \mathrm{d} t+\frac{1}{2} \sum_{\vec{q}} \iint \mathrm{d} t \mathrm{~d} s\left|\xi_{\vec{q}}\right|^{2} \mathrm{e}^{-i \vec{q} \cdot(\vec{r}(t)-\vec{r}(s))} \mathrm{e}^{-|t-s|} .
$$

Substituting for $\left|\xi_{\vec{q}}\right|^{2}$ and performing the summation over $\vec{q}$ using (27) we immediately get

$$
S=\int_{t_{a}}^{t_{b}}\left(-\frac{1}{2} \dot{\vec{r}}^{2}-V_{0} \mathrm{e}^{-r^{2} / 2 R^{2}}\right) \mathrm{d} t+\frac{\alpha}{2 \sqrt{ } 2} \iint \mathrm{d} t \mathrm{~d} s \frac{\mathrm{e}^{-|t-s|}}{|\vec{r}(t)-\vec{r}(s)|} .
$$

This action is however not path integrable and in the usual Feynman method one chooses a trial action which should contain the essential features of the actual action and should be path integrable. One may thus choose the following trial action

$$
S_{0}=\int_{t_{a}}^{t_{b}}\left(-\frac{1}{2} \dot{\vec{r}}^{2}-V_{0} \mathrm{e}^{-r^{2} / 2 R^{2}}\right) \mathrm{d} t-C \iint \mathrm{d} t \mathrm{~d} s[\vec{r}(t)-\vec{r}(s)]^{2} \mathrm{e}^{-w|t-s|} .
$$

It is well known that for the calculation of the GS energy one has to take the long-time limit to separate the GS from the excited states. Since in the long-time limit the above non-local Gaussian action may not differ much from the actual non-local Coulombic action, it would not be a bad approximation to treat (55) as a trial action and compensate for the errors introduced in the process by optimizing the values of the variational parameters $C$ and $w$. The palpable advantage with the action (55) is that it is exactly integrable. One then uses the Feynman variational principle

$$
E_{0, \text { exact }}^{\mathrm{ND}} \leq E_{F}^{\mathrm{ND}}=E_{0, \text { trial }}^{\mathrm{ND}}-s,
$$

where $E_{0 \text {,exact }}^{\mathrm{ND}}$ is the exact GS energy, i.e., the energy corresponding to the action $S, E_{0 \text {, trial }}^{\mathrm{ND}}$ is the GS energy corresponding to the path-integrable trial action $S_{0}$ and $s$ is given by

$$
s=\lim _{\left(t_{a}-t_{b}\right) \rightarrow \infty} \frac{\ll\left(S-S_{0}\right) \gg}{\left(t_{b}-t_{a}\right)},
$$

where

$$
\ll \ldots \gg=\frac{\int \ldots \mathrm{e}^{S_{0}} \mathrm{D} \vec{r}(t)}{\int \mathrm{e}^{S_{0}} \mathrm{D} \vec{r}(t)} .
$$

We shall however follow the Haken approach [22] of calculating the path integrals which is a convenient procedure for a bound state problem. In this approach we choose a general effective action in the beginning. For instance, we choose

$$
S_{\text {eff }}=\int_{t_{a}}^{t_{b}} L_{\text {eff }} \mathrm{d} t=\int_{t_{a}}^{t_{b}}\left[-\frac{1}{2} \dot{\vec{r}}^{2}-V_{\text {eff }}(\vec{r}(t))\right] \mathrm{d} t,
$$

where $V_{\text {eff }}$ is the effective potential which we shall specify later. The quantum mechanical hamiltonian $H_{\text {eff }}$ corresponding to the classical Lagrangian $L_{\text {eff }}$ satisfies the Schrödinger equation

$$
H_{\mathrm{eff}} \phi_{j, \mathrm{eff}}^{\mathrm{ND}}=E_{j, \mathrm{eff}}^{\mathrm{ND}} \phi_{j, \mathrm{eff}}^{\mathrm{ND}}
$$


where $\phi_{0 \text {, eff }}^{\mathrm{ND}}$ and $E_{0, \text { eff }}^{\mathrm{ND}}$ are respectively the GS wave function and the GS energy of $H_{\text {eff }}$. The effective transformation function corresponding to (59) can now be written as

$$
K_{\mathrm{eff}}\left(\vec{r}_{b}, t_{b} ; \vec{r}_{a}, t_{a}\right)=\int_{a}^{b} D \vec{r}(t) \mathrm{e}^{S_{\mathrm{eff}}},
$$

which can be expanded on the basis of the hamiltonian $H_{\mathrm{eff}}$ as

$$
K_{\mathrm{eff}}\left(\vec{r}_{b}, t_{b} ; \vec{r}_{a}, t_{a}\right)=\sum_{n} \phi_{n, \mathrm{eff}}^{\mathrm{ND}} \phi_{n, \mathrm{eff}}^{\mathrm{ND}} \mathrm{e}^{-E_{n, \mathrm{eff}}^{\mathrm{ND}\left(t_{b}-t_{a}\right)}} .
$$

The Feynman variational principle now reads

$$
E_{0, \text { exact }}^{\mathrm{ND}} \leq E_{F}^{\mathrm{ND}}=E_{0, \text { eff }}^{\mathrm{ND}}-s,
$$

where $s$ is given by

$$
s=\lim _{\left(t_{a}-t_{b}\right) \rightarrow \infty} \frac{\ll\left(S-S_{\mathrm{eff}}\right) \gg}{\left(t_{b}-t_{a}\right)} .
$$

$\ll\left(S-S_{\text {eff }}\right) \gg$ can be written as

$$
\ll S-S_{\text {eff }} \gg=\frac{\int\left(S-S_{\text {eff }}\right) \mathrm{e}^{S_{\text {eff }}} D \vec{r}(t)}{\int \mathrm{e}^{S_{\text {eff }} D \vec{r}(t)}}=B_{1}+B_{2},
$$

where $B_{1}$ and $B_{2}$ are given by

$$
B_{1}=\frac{\int_{a}^{b}\left(\int_{t_{a}}^{t_{b}}\left[-V_{0} \mathrm{e}^{-\frac{r^{2}}{2 R^{2}}}+V_{\text {eff }}(\vec{r})\right] \mathrm{d} t\right) \mathrm{e}^{S_{\text {eff }} D \vec{r}(t)}}{\int \mathrm{e}^{S_{\text {eff }} D \vec{r}}}
$$

and

$$
B_{2}=\frac{\int \sum\left|\xi_{q}\right|^{2} \iint \mathrm{d} s \mathrm{~d} t \mathrm{e}^{-i \vec{q} \cdot(\vec{r}(t)-\vec{r}(s))} \mathrm{e}^{-|t-s|} \mathrm{e}^{S_{\text {eff }}} D \vec{r}}{\int \mathrm{e}^{S_{\text {eff }} D \vec{r}}} .
$$

The numerator of $B_{1}$ can be written as

$$
\begin{aligned}
B_{1}^{\mathrm{num}}= & \int_{a}^{b} \mathrm{~d} \vec{r}_{t} \int_{t_{a}}^{t_{b}} \mathrm{~d} t K_{\mathrm{eff}}\left(\vec{r}_{t_{b}}, t ; \vec{r}_{t}, t\right) \\
& \times\left[-V_{0} \mathrm{e}^{-r_{t}^{2} / 2 R^{2}}+V_{\mathrm{eff}}\left(\vec{r}_{t}\right)\right] \times K_{\mathrm{eff}}\left(\vec{r}_{t}, t ; \vec{r}_{t_{a}}, t_{a}\right),
\end{aligned}
$$

which in the long-time limit reduces to

$$
\begin{aligned}
B_{1}^{\mathrm{num}} \approx & \int_{a}^{b} \mathrm{~d} \vec{r}_{t} \int_{t_{a}}^{t_{b}} \mathrm{~d} t \phi_{0, \mathrm{eff}}^{\mathrm{ND}}\left(\vec{r}_{t_{b}}\right) \phi_{0, \mathrm{eff}}^{\mathrm{ND} *}\left(\vec{r}_{t}\right) \times \mathrm{e}^{-E_{0, \text { eff }}^{\mathrm{ND}}\left(t_{b}-t\right)} \times\left[-V_{0} \mathrm{e}^{-r_{t}^{2} / 2 R^{2}}+V_{\mathrm{eff}}\left(\vec{r}_{t}\right)\right] \\
& \times \phi_{0, \text { eff }}^{\mathrm{ND}}\left(\vec{r}_{t}\right) \phi_{0, \text { eff }}^{\mathrm{ND} *}\left(\vec{r}_{t_{a}}\right) \times \mathrm{e}^{-E_{0, \mathrm{eff}}^{\mathrm{ND}}\left(t-t_{a}\right)} .
\end{aligned}
$$

Integration over $t$ can be done trivially to give

$$
\begin{aligned}
B_{1}^{\mathrm{num}} \approx & \left(t_{b}-t_{a}\right) \phi_{0, \mathrm{eff}}^{\mathrm{ND}}\left(\vec{r}_{t_{b}}\right) \phi_{0, \mathrm{eff}}^{\mathrm{ND} *}\left(\vec{r}_{t_{a}}\right) \times \mathrm{e}^{-E_{0, \mathrm{eff}}^{\mathrm{ND}}\left(t_{b}-t_{a}\right)} \int \mathrm{d} \vec{r}_{t} \phi_{0, \mathrm{eff}}^{\mathrm{ND} *}\left(\vec{r}_{t}\right) \\
& \times\left[-V_{0} \mathrm{e}^{-r_{t}^{2} / 2 R^{2}}+V_{\mathrm{eff}}\left(\vec{r}_{t}\right)\right] \times \phi_{0, \mathrm{eff}}^{\mathrm{ND}}\left(\vec{r}_{t}\right)
\end{aligned}
$$


Although the quantity $\left[-V_{0} \mathrm{e}^{-r_{t}^{2} / 2 R^{2}}+V_{\text {eff }}\left(\vec{r}_{t}\right)\right]$ is a function of the classical variable $r$, the integral in the above equation can be considered as the expectation value of the quantum mechanical operator $\left[-V_{0} \mathrm{e}^{-r_{t}^{2} / 2 R^{2}}+V_{\text {eff }}\left(\vec{r}_{t}\right)\right]$ with respect to the wave function $\phi_{0, \text { eff }}^{\mathrm{ND}}\left(\vec{r}_{t}\right)$. Thus we can write

$$
\begin{aligned}
B_{1}^{\mathrm{num}} \approx & \left(t_{b}-t_{a}\right) \phi_{0, \mathrm{eff}}^{\mathrm{ND}}\left(\vec{r}_{t_{b}}\right) \phi_{0, \mathrm{eff}}^{\mathrm{ND} *}\left(\vec{r}_{t_{a}}\right) \mathrm{e}^{-E_{0, \mathrm{eff}}^{\mathrm{ND}}\left(t_{b}-t_{a}\right)} \\
& \times\left\langle\phi_{0, \mathrm{eff}}^{\mathrm{ND} *}\left|-V_{0} \mathrm{e}^{-r_{t}^{2} / 2 R^{2}}+V_{\mathrm{eff}}\left(\vec{r}_{t}\right)\right| \phi_{0, \mathrm{eff}}^{\mathrm{ND}}\right\rangle .
\end{aligned}
$$

In the same limit, the denominator of (64) reduces to

$$
B_{1}^{\mathrm{den}} \approx \phi_{0, \text { eff }}^{\mathrm{ND}}\left(\vec{r}_{t_{b}}\right) \phi_{0, \text { eff }}^{\mathrm{ND} *}\left(\vec{r}_{t_{a}}\right) \mathrm{e}^{-E_{0, \mathrm{eff}}^{\mathrm{ND}}\left(t_{b}-t_{a}\right)} .
$$

Therefore, $B_{1}$ simplifies to

$$
B_{1} \approx\left(t_{b}-t_{a}\right)\left\langle\phi_{0, \text { eff }}^{\mathrm{ND} *}\left|-V_{0} \mathrm{e}^{-r^{2} / 2 R^{2}}+V_{\mathrm{eff}}(\vec{r})\right| \phi_{0, \mathrm{eff}}^{\mathrm{ND}}\right\rangle .
$$

In a similar way one can show that

$$
B_{2}=-\left(t_{b}-t_{a}\right) \sum_{[j], \vec{q}} \frac{\left|\left\langle\Phi_{[j], \mathrm{eff}}\left|\xi_{\vec{q}} \mathrm{e}^{-i \vec{q} \cdot \vec{r}}\right| \Phi_{0, \mathrm{eff}}\right\rangle\right|^{2}}{E_{[j], \mathrm{eff}}-E_{[0], \mathrm{eff}}+1}
$$

Eq. (56) then becomes

$$
E_{0, \text { exact }}^{\mathrm{ND}} \leq E_{\mathrm{FH}}^{\mathrm{ND}}=T_{1}+T_{2},
$$

where

$$
T_{1}=\left\langle\phi_{0, \text { eff }}^{\mathrm{ND}}\left|\left(-\frac{1}{2} \vec{\nabla}_{\vec{r}}^{2}-V_{0} \mathrm{e}^{-\frac{r^{2}}{2 R^{2}}}\right)\right| \phi_{0, \text { eff }}^{\mathrm{ND}}\right\rangle,
$$

and

$$
T_{2}=-\sum_{[j], \vec{q}} \frac{\left|\left\langle\Phi_{[j], \text { eff }}\left|\xi_{\vec{q}} \mathrm{e}^{-i \vec{q} \cdot \vec{r}}\right| \Phi_{0, \mathrm{eff}}\right\rangle\right|^{2}}{E_{[j], \mathrm{eff}}-E_{[0], \mathrm{eff}}+1}
$$

To proceed further, now we have to give a prescription for the trial functions, $\Phi_{[j], \text { eff }}(\vec{r})$ or in other words we have to choose the potential, $V_{\mathrm{eff}}(\vec{r})$. We shall work in the harmonic oscillator approximation and therefore choose

$$
V_{\mathrm{eff}}(\vec{r})=\frac{1}{2} \omega^{2} r^{2}
$$

The corresponding wave functions $\Phi_{[j], \text { eff }}(\vec{r})$ and the energy spectrum $E_{[j] \text {, eff }}$ are then given by Eqs. (9) and (10) respectively with $\tilde{\omega}_{h}$ replaced by $\omega$. The Feynman-Haken variational energy finally assumes the following expression

$$
E_{\mathrm{FH}}^{\mathrm{ND}}=\frac{N}{4}(2-\lambda) \omega-\frac{\lambda V_{0}}{\left(1+\frac{1}{2 \omega R^{2}}\right)}-\left(\frac{\alpha \sqrt{\pi}}{2 \sqrt{\omega}}\right)\left[\frac{\Gamma\left(\frac{N-1}{2}\right)}{\Gamma\left(\frac{N}{2}\right)}\right]\left[\frac{\Gamma\left(\frac{1}{\omega}\right)}{\Gamma\left(\frac{1}{\omega}+\frac{1}{2}\right)}\right] .
$$


The above expression has to be minimized with respect to $\omega$ for $N=2$ and $N=3$ separately and the polaronic correction, $\Delta E_{\mathrm{FH}}$ is then obtained as

$$
\Delta E_{\mathrm{FH}}^{\mathrm{ND}}=E_{\mathrm{FH}}^{\mathrm{ND}}(\alpha)-E_{\mathrm{FH}}^{\mathrm{ND}}(\alpha=0)=-\left(\frac{\alpha \sqrt{\pi}}{2 \sqrt{\omega}}\right)\left[\frac{\Gamma\left(\frac{N-1}{2}\right)}{\Gamma\left(\frac{N}{2}\right)}\right]\left[\frac{\Gamma\left(\frac{1}{\omega}\right)}{\Gamma\left(\frac{1}{\omega}+\frac{1}{2}\right)}\right] .
$$

The numerical results will be discussed in Section 4. One can of course obtain a few interesting limiting cases. But we shall not report any limiting results in this case, because almost similar limiting results have already been presented while discussing the RSPT results.

\subsection{Lee-Low-Pines-Huybrechts (LLPH) method}

In the previous section we have discussed the Feynman-Haken (FH) path-integral method which provides solutions for the entire range of the coupling parameters and also yields the lowest upper bounds. However the disadvantage with this method is that it cannot be applied to the excited states, for it uses the Feynman-Jensen inequality and the long-time limit in which case, only the GS gets separated from the other excited states for a discrete energy spectrum. Furthermore, this method is also not so useful for the calculation of quantities like the number of phonons in the polaron, the polaron size, the polarization potential and so on because these require the knowledge of the wave function and this method is particularly based on the Lagrangian. Therefore in the present section, we shall look for an all-coupling variational solution of (3) and use a variant of the celebrated Lee-Low-Pines (LLP) transformation method [23], namely the Lee-Low-Pines-Huybrechts (LLPH) technique [24]. Though this method is inferior to the Feynman path-integral approach (FPIA) in terms of accuracy, it has the advantage in that it gives the wave function for the problem being a Ritz variational technique and thus can describe various polaronic properties. In the LLPH method we successively apply two unitary transformations with the operators

$$
U_{1}=\mathrm{e}^{S_{1}}=\exp \left[-i a \sum_{\vec{q}}(\vec{q} \cdot \vec{r}) b_{\vec{q}}^{\dagger} b_{\vec{q}}\right]
$$

and

$$
U_{2}=\mathrm{e}^{S_{2}}=\exp \left[\sum_{\vec{q}}\left(f_{\vec{q}} b_{\vec{q}}^{\dagger}-f_{\vec{q}}^{*} b_{\vec{q}}\right)\right]
$$

where $a$ and $f_{\vec{q}}$ are variational parameters and then perform a zero-phonon averaging to obtain an effective electronic hamiltonian, the expectation value of which with respect to a suitable electronic function is then minimized with respect to the variational parameters. This procedure is equivalent to choosing a trial variational wave function as

$$
|\Psi\rangle=\mathrm{e}^{\left(-i a \sum_{\vec{q}}(\vec{q} \cdot \vec{r}) b_{\vec{q}}^{\dagger} b_{\vec{q}}\right)} \times \mathrm{e}^{\left(\sum_{\vec{q}}\left(f_{\vec{q}} b_{\vec{q}}^{\dagger}-f_{\vec{q}}^{*} b_{\vec{q}}\right)\right)}|0\rangle|\Phi(\vec{r})\rangle,
$$

where $|0\rangle$ is the unperturbed zero-phonon state defined in Eq. (14) and $\Phi(\vec{r})$ is the electronic wave function that will be chosen later. The energy is of course given by $E=\langle\Psi|H| \Psi\rangle$. This procedure reduces to the LLP method if we take $a=1$, which provides a good description in the 
extended state limit. In the other limit i.e., in the adiabatic case or the localized state limit, one chooses $a=0$, which is as expected equivalent to the Landau-Pekar method [18]. By treating $a$ as a variational parameter encompassing the range $(0,1)$, one can thus have a good description for the entire coupling parameter space. The energy can be written as

$$
E=\langle 0|\langle\Psi|H| \Psi\rangle| 0\rangle=\langle\Phi(\vec{r})|\langle 0|\tilde{\tilde{H}}| 0\rangle| \Phi(\vec{r})\rangle,
$$

where

$$
\tilde{\tilde{H}}=U_{2}^{-1} U_{1}^{-1} H U_{1} U_{2}=U_{2}^{-1} \tilde{H} U_{2} .
$$

After some algebraic manipulation, we obtain

$$
\begin{aligned}
\tilde{\tilde{H}}= & \frac{\vec{p}^{2}}{2}-V_{o} \mathrm{e}^{-r^{2} / 2 R^{2}}+\sum_{\vec{q}}\left(1-a \vec{p} \cdot \vec{q}+\frac{a^{2} q^{2}}{2}\right)\left(b_{\vec{q}}^{+}+f_{\vec{q}}^{*}\right)\left(b_{\vec{q}}+f_{\vec{q}}\right) \\
& +\frac{a^{2}}{2} \sum_{\vec{q}, \vec{q}^{\prime}} \vec{q} \cdot \vec{q}^{\prime}\left(b_{\vec{q}}^{+}+f_{\vec{q}}^{*}\right)\left(b_{\vec{q}^{\prime}}^{+}+f_{\vec{q}^{\prime}}^{*}\right)\left(b_{\vec{q}}+f_{\vec{q}}\right)\left(b_{\vec{q}^{\prime}}+f_{\vec{q}^{\prime}}\right) \\
& +\sum_{\vec{q}}\left[\xi_{\vec{q}} \mathrm{e}^{-(1-a) \vec{q} \cdot \vec{r}}\left(b_{\vec{q}}^{+}+f_{\vec{q}}^{*}\right)+\text { h.c. }\right] .
\end{aligned}
$$

The effective electronic hamiltonian becomes, after eliminating the phonon degrees of freedom by zero-phonon averaging,

$$
\begin{aligned}
H_{\mathrm{el}}^{\mathrm{eff}}= & \langle 0|\tilde{\tilde{H}}| 0\rangle=\frac{\vec{p}^{2}}{2}-V_{o} \mathrm{e}^{-r^{2} / 2 R^{2}}+\frac{a^{2}}{2}\left[\sum_{\vec{q}} \vec{q}\left|f_{\vec{q}}\right|^{2}\right]^{1 / 2} \\
& +\sum_{\vec{q}}\left(1-a \vec{p} \cdot \vec{q}+\frac{a^{2} q^{2}}{2}\right)\left|f_{\vec{q}}\right|^{2}+\sum_{\vec{q}}\left[\xi_{\vec{q}} \mathrm{e}^{-(1-a) \vec{q} \cdot \vec{r}} f_{\vec{q}}^{*}+h . c .\right] .
\end{aligned}
$$

The LLPH variational energy is now given by

$$
\begin{aligned}
E_{\mathrm{LLPH}}= & \left\langle\Phi\left|H_{\mathrm{el}}^{\mathrm{eff}}\right| \Phi\right\rangle=\frac{1}{2}\left\langle\Phi\left|\vec{p}^{2}\right| \Phi\right\rangle-V_{o}\left\langle\Phi\left|\mathrm{e}^{-r^{2} / 2 R^{2}}\right| \Phi\right\rangle \\
& +\sum_{\vec{q}}\left(1-a \vec{q} \cdot\langle\Phi|\vec{p}| \Phi\rangle+\frac{a^{2} q^{2}}{2}\right)\left|f_{\vec{q}}\right|^{2}+\frac{a^{2}}{2}\left[\sum_{\vec{q}} \vec{q}\left|f_{\vec{q}}\right|^{2}\right]^{1 / 2} \\
& +\sum_{\vec{q}}\left[\xi_{\vec{q}}\left\langle\Phi\left|\mathrm{e}^{-(1-a) \vec{q} \cdot \vec{r}}\right| \Phi\right\rangle f_{\vec{q}}^{*}+\text { c.c. }\right] .
\end{aligned}
$$

Minimization of $E_{\mathrm{LLPH}}$ with respect to $f_{\vec{q}}^{*}$ yields

$$
f_{\vec{q}}^{*}=-\frac{\xi_{\vec{q}} \rho_{\vec{q}}^{*}}{1-a\langle\Phi|\vec{p}| \Phi\rangle \cdot \vec{q}+\frac{a^{2} q^{2}}{2}},
$$

where

$$
\rho_{\vec{q}}=\left\langle\Phi\left|\mathrm{e}^{-(1-a) \vec{q} \cdot \vec{r}}\right| \Phi\right\rangle,
$$


and we have stipulated the condition

$$
\sum_{\vec{q}} \vec{q}\left|f_{\vec{q}}\right|^{2}=0
$$

which is a reasonable approximation for a symmetric dot. For a symmetric dot, we also have, $\langle\Phi|\vec{p}| \Phi\rangle=0$ and so the Eqs. (89) and (88) reduce to

$$
f_{\vec{q}}=-\frac{\xi_{\vec{q}} \rho_{\vec{q}}^{*}}{1+\frac{a^{2} q^{2}}{2}}
$$

and

$$
E_{\mathrm{LLPH}}=\frac{1}{2}\left\langle\Phi\left|\vec{p}^{2}\right| \Phi\right\rangle-V_{o}\left\langle\Phi\left|\mathrm{e}^{-r^{2} / 2 R^{2}}\right| \Phi\right\rangle+\sum_{\vec{q}} \frac{\left|\xi_{\vec{q}}\right|^{2}\left|\rho_{\vec{q}}\right|^{2}}{1+\frac{a^{2} q^{2}}{2}} .
$$

We would also like to study a few other properties of the polaron problem in the present case.

For example, the number of phonons in the phonon cloud around the polaron can be defined as

$$
N_{\text {pol }}=\left\langle\Psi\left|\sum_{\vec{q}} b_{\vec{q}}^{\dagger} b_{\vec{q}}\right| \Psi\right\rangle,
$$

which on substituting (83), leads, after some calculation, to

$$
N_{\mathrm{pol}}^{\mathrm{ND}}=\sum_{\vec{q}} \frac{\left|\xi_{\vec{q}}\right|^{2}\left|\rho_{\vec{q}}\right|^{2}}{\left(1+\frac{a^{2} q^{2}}{2}\right)^{2}}
$$

We next consider the polaron size. The polaron size can of course be defined in various ways. All the definitions give qualitatively the similar behaviour and the same order of magnitudes. Here we shall follow the simplest definition:

$$
R_{\mathrm{pol}}=\langle\Psi|r| \Psi\rangle .
$$

Finally, we consider the polarization potential which can be defined as

$$
V_{\mathrm{pol}}\left(\vec{r}^{\prime}\right)=\left\langle\Psi\left|v\left(\vec{r}-\vec{r}^{\prime}\right)\right| \Psi\right\rangle
$$

where

$$
v(\vec{r})=-\frac{1}{e} \sum_{\vec{q}}\left(\xi_{\vec{q}} \mathrm{e}^{-i \vec{q} \cdot \vec{r}} b_{\vec{q}}^{\dagger}+\text { h.c. }\right) .
$$

We obtain

$$
-e V_{\mathrm{pol}}(\vec{r})=-2 \sum_{\vec{q}} \frac{\left|\xi_{\vec{q}}\right|^{2}\left|\rho_{\vec{q}}\right|^{2}}{1+\frac{a^{2} q^{2}}{2}} \cos (\vec{q} \cdot \vec{r}) .
$$

We have not yet specified the form of the electronic function. We are interested here in the ground state of the problem for which we choose the electronic function $\Phi(\vec{r})$ in the harmonic oscillator 
approximation in $N$-dimensions as

$$
\left|\Phi_{\mathrm{GS}}\right\rangle=\left(\frac{\delta^{N}}{\pi^{N / 2}}\right)^{1 / 2} \mathrm{e}^{-\left(\delta^{2} r^{2} / 2\right)}
$$

where $\delta$ is a variational parameter. We then obtain

$$
\rho_{\vec{q}}=\mathrm{e}^{-\frac{(1-a)^{2}}{4 \delta^{2}} q^{2}} .
$$

The GS energy now reads

$$
E_{\mathrm{LLPH}}^{\mathrm{ND}}=\frac{N}{4 \delta^{2}}-V_{o}\left[1+\frac{\beta^{2}}{\delta^{2}}\right]^{-N / 2}-\frac{\alpha \sqrt{\pi}}{2} \frac{\Gamma\left(\frac{N-1}{2}\right)}{\Gamma\left(\frac{N}{2}\right)}(1+\delta t) \mathrm{e}^{t^{2}} \operatorname{erfc}(t),
$$

where we have defined a new variational parameter $t$ instead of $a$, which is given by $t=$ $(1-a) a / \delta$. The other quantities then assume the following expressions:

$$
\begin{aligned}
& N_{\mathrm{pol}, \mathrm{LLPH}}^{\mathrm{ND}}=\frac{\alpha}{\sqrt{2 \pi}} \frac{\Gamma\left(\frac{N-1}{2}\right)}{\Gamma\left(\frac{N}{2}\right)} \int_{0}^{\infty} \mathrm{d} q \frac{\mathrm{e}^{-\frac{(1-a)^{2} q^{2}}{2 \delta^{2}}}}{\left(1+\frac{a^{2} q^{2}}{2}\right)^{2}}, \\
& R_{\mathrm{pol}, \mathrm{LLPH}}^{\mathrm{ND}}=\frac{\Gamma\left(\frac{N-1}{2}\right)}{\Gamma\left(\frac{N}{2}\right)} \frac{1}{\delta} \\
& -e V_{\mathrm{pol}, \mathrm{LLPH}}^{\mathrm{ND}}(\vec{r})=-\left(\frac{2^{\frac{N-1}{2}} \Gamma\left(\frac{N-1}{2}\right)}{\sqrt{\pi}}\right)\left(\frac{\alpha}{r\left(\frac{N}{2}-1\right)}\right) \\
& \times \int_{0}^{\infty} \mathrm{d} q \frac{\mathrm{e}^{-\frac{(1-a)^{2} q^{2}}{2 \delta^{2}}}}{q^{\left(\frac{N}{2}-1\right)}\left(1+\frac{a^{2} q^{2}}{2}\right)} \times J_{\frac{N}{2}-1}(q r) .
\end{aligned}
$$

Variation of (102) with respect to $\delta$ and $t$ gives

$$
\delta^{4}-\left[\frac{\alpha \sqrt{\pi}}{2} \frac{\Gamma\left(\frac{N-1}{2}\right)}{\Gamma\left(\frac{N}{2}\right)} t \mathrm{e}^{t^{2}} \operatorname{erfc}(t)\right] \delta^{3}-2 V_{o} \beta^{2}\left(1+\frac{\beta^{2}}{\delta^{2}}\right)^{-\left(\frac{N}{2}+1\right)}=0
$$

and

$$
2(1+t \delta)+\sqrt{\pi} \mathrm{e}^{t^{2}} \operatorname{erfc}(t)(\delta+2 \sqrt{\pi}(1+t \delta))=0
$$

which have to be solved numerically for $N=2$ and $N=3$. The numerical results will be presented in Section 4 to which we turn now.

\section{Numerical results and discussion}

For numerical computations we have to specify the value of $N$. We are interested here in both $2 \mathrm{D}$ and $3 \mathrm{D}$ dots and therefore we perform numerical computation for $N=2$ and $N=3$. The 


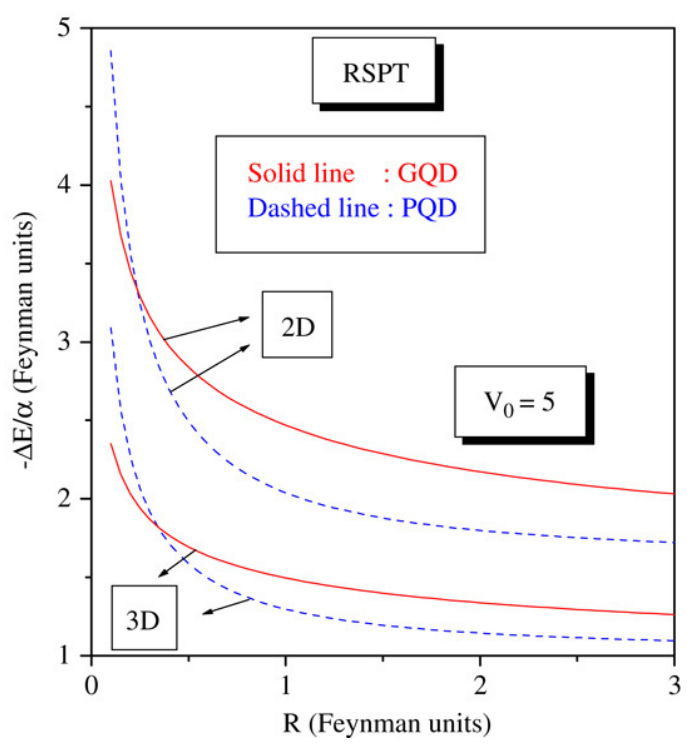

Fig. 1. Polaron self-energy in Feynman units as a function of the range of the confining Gaussian potential, $R$ for $V_{0}=20$ and $V_{0}=30$ for a parabolic quantum dot and also for a Gaussian quantum dot in both 2D and 3D. The solid line refers to the Gaussian potential while the dashed line describes the parabolic confinement case.

polaron self-energies according to the RSPT approach then assume the following expressions:

$$
-\frac{\Delta E_{\mathrm{RSPT}}}{\alpha}=\sqrt{\omega_{h}}\left[\frac{\Gamma\left(\frac{1}{\omega_{h}}+1\right)}{\Gamma\left(\frac{1}{\omega_{h}}+\frac{1}{2}\right)}\right], \quad \text { in } 3 D,
$$

and

$$
-\frac{\Delta E_{\mathrm{RSPT}}}{\alpha}=\frac{\pi}{2} \sqrt{\omega_{h}}\left[\frac{\Gamma\left(\frac{1}{\omega_{h}}+1\right)}{\Gamma\left(\frac{1}{\omega_{h}}+\frac{1}{2}\right)}\right], \quad \text { in } 2 D .
$$

First we make a comparative study of the Gaussian confinement vis-a-vis the parabolic confinement for two values of $V_{0}$, namely, $V_{0}=20$ and $V_{0}=30$ for both 2D and 3D systems. For the case of a parabolic quantum dot, $\omega_{h}$ is replaced by $\tilde{\omega}_{h}$. The results are shown in Fig. 1. One can observe that there is a sizable difference between the results obtained from the two potentials. It is clear that for larger confinement lengths, the parabolic potential underestimates the polaronic corrections. The discrepancy apparently increases with increasing $V_{0}$. For small confinement lengths, on the other hand, we find, rather surprisingly, that the parabolic potential overestimates the polaronic correction and in $2 \mathrm{D}$, the discrepancy is ever larger. Thus there is a crossing of energy curves at small values of $R$. This apparently intriguing behaviour can however be understood from the limiting result (44) which says that in the strong-confinement limit, the polaron self-energy is inversely proportional to $l$ and we observe that in the same limit, $l$ can be larger for a Gaussian quantum dot than for a corresponding parabolic quantum dot leading to a smaller polaron self-energy for a GQD than for a PQD. The difference in the $l$-value between a GQD and a PQD is rather small in $3 \mathrm{D}$, but in $2 \mathrm{D}$ it is quite large and therefore polaronic 


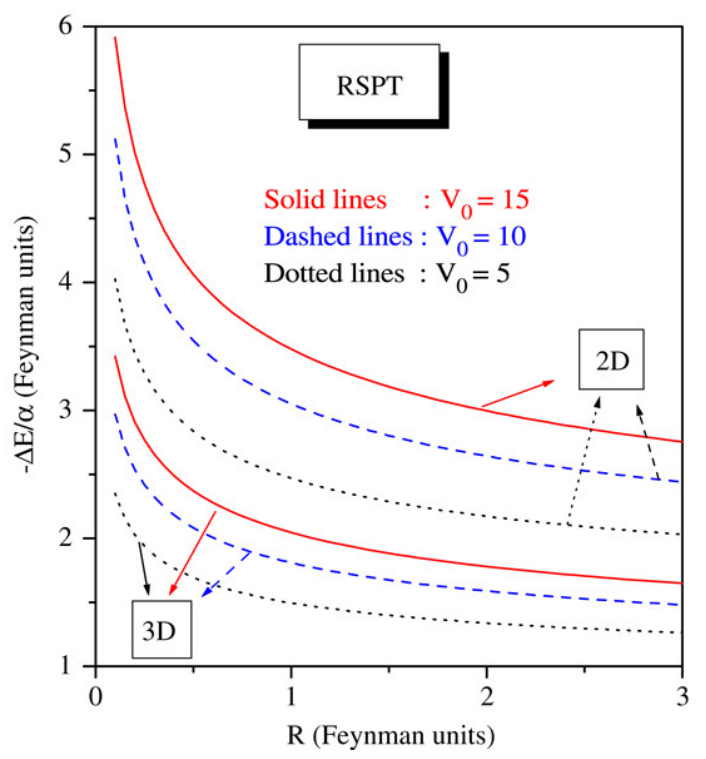

Fig. 2. Polaron self-energy, $-\Delta E / \alpha$ in Feynman units as a function of $R$ for three values of the depth of the Gaussian confinement potential, $V_{0}=10,20,30$ in both $2 \mathrm{D}$ and $3 \mathrm{D}$.

effect turns out to be much smaller in a 2D Gaussian dot as compared to that in a parabolic dot as $R$ becomes small. Thus we conclude that for a quantum dot, parabolic potential is only a poor approximation of the more realistic Gaussian confining potential for the entire range of the confinement length.

In Fig. 2, we study the polaronic correction to the GS energy obtained from RSPT as a function of the range of the potential $R$ for three values of the depth of the Gaussian potential, $V_{0}$, both for 2D and 3D systems. It is evident that the polaronic effect increases as the depth of the potential increases. The polaronic correction being linear in $\alpha$ in RSPT, it is apparent that the electron-phonon interaction and the confining potential work synergistically. Comparison between the 2D and 3D curves shows that the qualitative behaviour in $2 \mathrm{D}$ is the same as that in $3 \mathrm{D}$ but the polaronic effect is much stronger in two dimensions. This is of course consistent with the common notion that polaronic effect decreases with increasing dimensionality.

Next we would like to compare the results obtained from the three different methods. For the Feynman-Haken results, we have to consider (79) with $\lambda=1$. The GS Feynman-Haken energies for 2D and 3D Gaussian quantum dots are given respectively by

$$
E_{\mathrm{FH}}^{2 D}=\frac{1}{2 \mu^{2}}-\frac{V_{0}}{\left(1+\frac{\mu^{2}}{2 R^{2}}\right)}-\left(\frac{\alpha \pi}{2 \mu}\right) \frac{\Gamma\left(\mu^{2}+1\right)}{\Gamma\left(\mu^{2}+\frac{1}{2}\right)},
$$

and

$$
E_{\mathrm{FH}}^{3 D}=\frac{3}{2 \mu^{2}}-\frac{V_{0}}{\left(1+\frac{\mu^{2}}{2 R^{2}}\right)^{3 / 2}}-\left(\frac{\alpha \pi}{2 \mu}\right) \frac{\Gamma\left(\mu^{2}+1\right)}{\Gamma\left(\mu^{2}+\frac{1}{2}\right)},
$$

where $\mu^{2}=1 / \omega$. Eqs. (110) and (111) are minimized with respect to $\mu$ both in the presence of the electron-phonon interaction and also for $\alpha=0$. The polaron self-energies are then obtained 


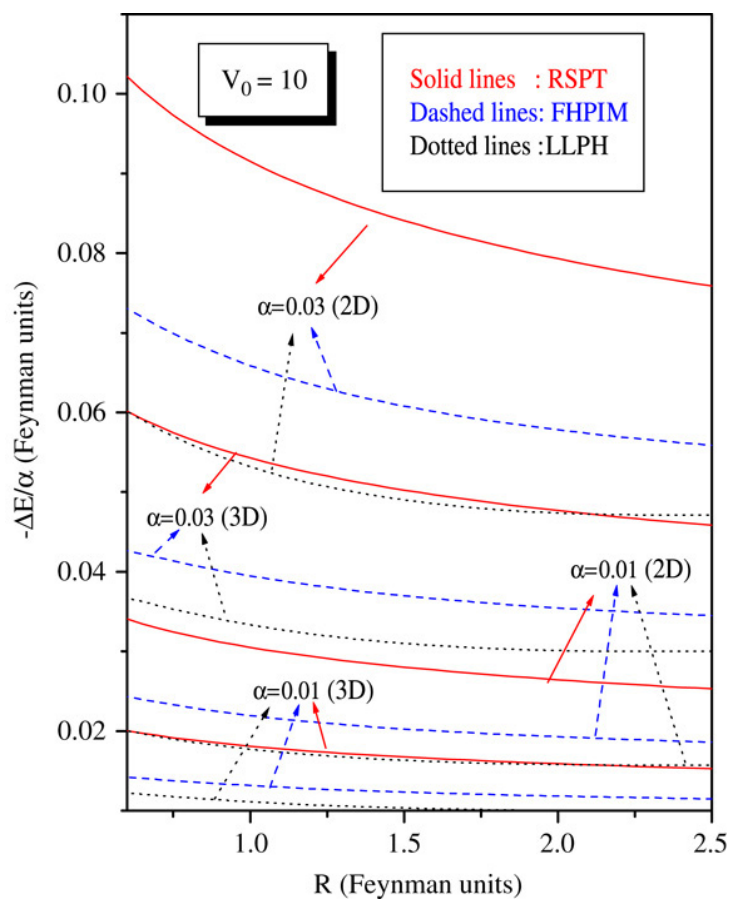

Fig. 3. Polaron self-energy in Feynman units as a function of the range $R$ for $V_{0}=10$ and for three values of $\alpha$, $\alpha=0.01,0.02,0.03$ in 2D and 3D Gaussian quantum dots. The solid lines refer to the RSPT results, the dashed lines give the FHPIM results and the dotted lines represent the LLPH data.

using Eq. (80) for $N=2$ and $N=3$. To obtain the LLPH results we write the LLPH GS energy for two and three dimensions from (102):

$$
\begin{aligned}
& E_{\mathrm{LLPH}}^{2 D}=\frac{1}{2} \delta^{2}-\frac{V_{0}}{\left(1+\frac{1}{2 R^{2} \delta^{2}}\right)}-\frac{\alpha \pi}{2}(1+\delta t) \mathrm{e}^{t^{2}} \operatorname{erfc}(t) \\
& E_{\mathrm{LLPH}}^{3 D}=\frac{3}{4} \delta^{2}-\frac{V_{0}}{\left(1+\frac{1}{2 R^{2} \delta^{2}}\right)^{3 / 2}}-\alpha(1+\delta t) \mathrm{e}^{t^{2}} \operatorname{erfc}(t) .
\end{aligned}
$$

The above expressions are to be numerically minimized with respect to two variational parameters $\delta$ and $t$ to obtain the upper bound to the GS energy. $V_{0}=0$ of course yields the bulk polaron results. To obtain the polaronic corrections we then put $\alpha=0$ in (112) and (113) and minimize the corresponding expressions and then subtract the resulting values respectively from (112) and (113). In Fig. 3 we present the variation of the polaron self-energy as a function of $R$ for the weak-coupling range both for $2 \mathrm{D}$ and $3 \mathrm{D}$ quantum dots. It is of course evident from the results of all the three methods that as $R$ decreases, the polaronic effect becomes more and more pronounced and as expected, the polaron self-energy also increases with the electron-phonon coupling constant, $\alpha$. On the other hand, as the range of the potential increases substantially, the polaron self-energy saturates to a constant which is the bulk limit. One can again see that the polaronic effect is much stronger in $2 \mathrm{D}$ than in $3 \mathrm{D}$. These results are along the expected line. But certain other issues are worth pointing out. For example, it is quite interesting to note that RSPT 
provides the lowest results, the LLPH results are much higher than expected and FHPIM yields energy values that lie somewhere in between, to be more specific, a bit closer to the RSPT curves. In the case of the parabolic quantum dot, it is well known that in the weak-coupling regime all the three methods yield more or less the same results. In fact, for certain values of $\alpha$, the LLPH results may be even a little lower than those obtained from RSPT and FHPIM whose data fall almost on the same curve in the weak-coupling regime. The inaccuracy of the LLPH results in this work may not be difficult to trace. In fact it should not be considered as a shortcoming of this method, but is actually a consequence of the use of a too simplistic trial wave function for the electronic part. A better wave function incorporating the effect of anharmonicity of the confining potential will certainly improve the situation. Regarding the efficacy of the RSPT, one may of course argue that in view of the small values of the coupling constant considered, the success of the RSPT is but natural, but the point is that the RSPT results are far too lower than the FHPIM values which at the very first glance appears rather intriguing. However a careful look seems to justify this rather unusual behaviour. We believe that this is precisely because of the special partitioning of the Gaussian potential implemented in our perturbation theory and the subsequent use of the mean-field treatment for the unperturbed part. We have assumed that we can approximate the Gaussian potential

$$
V(r)=-V_{o} \mathrm{e}^{-r^{2} / 2 R^{2}}
$$

by

$$
V(r)=-V_{o}+\left\langle\Phi_{o}(\vec{r})\left|\frac{V_{o}}{r^{2}}\left(1-\mathrm{e}^{-r^{2} / 2 R^{2}}\right)\right| \Phi_{o}(\vec{r})\right\rangle r^{2}
$$

which essentially means that we have neglected a contribution which is of the order of

$$
\begin{aligned}
\Delta V= & \left\langle\Phi_{o}^{\prime}(\vec{r})\left|\frac{1}{r^{2}}\left(1-\mathrm{e}^{-r^{2} / 2 R^{2}}\right) r^{2}\right| \Phi_{o}^{\prime}(\vec{r})\right\rangle-\left\langle\Phi_{o}^{\prime}(\vec{r})\left|\frac{\left(1-\mathrm{e}^{-r^{2} / 2 R^{2}}\right)}{r^{2}}\right| \Phi_{o}^{\prime}(\vec{r})\right\rangle \\
& \times\left\langle\Phi_{o}^{\prime}(\vec{r})\left|r^{2}\right| \Phi_{o}^{\prime}(\vec{r})\right\rangle,
\end{aligned}
$$

where $\Phi_{o}^{\prime}(\vec{r})$ is the GS wave function for the renormalized (effective) harmonic oscillator of frequency $\omega_{h}$. Since, the right-hand side is always a positive quantity, the neglected quantity in our case is a positive quantity. Thus what we have done is a Hartree-Fock-like mean-field approximation and as a result we have obtained a lower energy for the Gaussian potential than what would be obtained had we used the harmonic oscillator wave function corresponding to the frequency $\tilde{\omega}_{h}$. Since the second-order RSPT correction due to the electron-phonon interaction has been obtained using this renormalized basis corresponding to $\omega_{h}$, it is quite natural that our RSPT result for the polaronic correction may lie lower than the conventional upper bound. It may be pointed out here that normally for the bulk bound polaron problem and for the parabolic quantum dot problem as well, the RSPT gives an upper bound to the GS polaron self-energy. However in the present case it is not clear whether we really get a lower bound or still an upper bound. All we can safely say is that the energy for the Gaussian potential obtained in the present method is lower than what would have been obtained using the unperturbed harmonic oscillator basis. However the partitioning of the Gaussian potential can be continued iteratively till we get a self-consistent result.

Of course, for smaller values of $\alpha$, the differences in the energies obtained from the three different methods are not very significant, but even for reasonable values of $\alpha$ the differences are too large to be ignored. In our subsequent discussion we shall consider the FH results as 


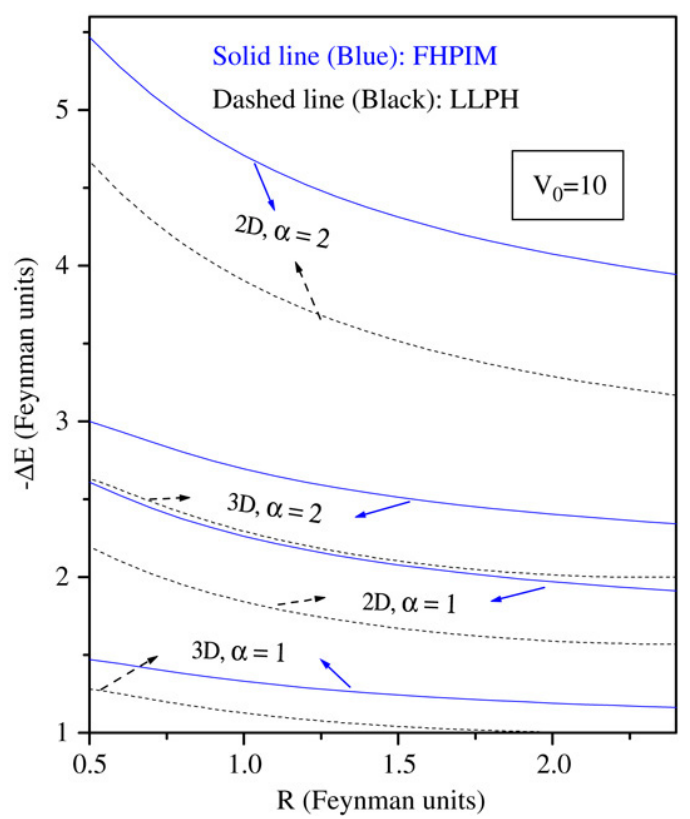

Fig. 4. Polaron self-energy, $-\Delta E$ in Feynman units obtained from the LLPH method and FHPIM as a function of $R$ for two values of $\alpha, \alpha=1$ and $\alpha=2$ and for $V_{0}=10$ in both 2D and 3D.

the reference upper bound for comparison, keeping of course in mind that the LLPH values are plagued with the problem rooted in the simplistic choice of the trial wave function.

In Fig. 4, we compare the Feynman-Haken energies with the LLPH values for the intermediate-coupling range as functions of $R$ for 3D and 2D quantum dots. Obviously the Feynman-Haken results turn out to be superior to the LLPH energies, as expected, but again the difference is much larger because of the reason mentioned in the preceding paragraph. In $3 \mathrm{D}$ the difference is a little smaller and so is the case for smaller $\alpha$ values. Nevertheless, the LLPH results give qualitatively the similar results and in the absolute scale their accuracy may be considered fairly alright. Again the polaronic effects are observed to be stronger in $2 \mathrm{D}$ than in 3D. In all the cases, however, for large $R$, the polaron self-energy saturates to some asymptotic value depending on the value of $\alpha$. These are the bulk limits. In Fig. 5, we next plot the strong-coupling results obtained from FHPIM and the LLPH method. We have shown the results for $\alpha=7$. One can see that the relative accuracy of the LLPH results for the strong-coupling case are much better. Thus the LLPH results, though may not give very accurate results in different regimes, can still be considered as an all-coupling method which is reasonably alright for the entire range of the different parameter values. And of course the situation can be further improved by choosing a better trial function. In Fig. 6, we compare the results obtained from all the three methods for different values of $V_{0}$ for a particular value of $\alpha$, namely, $\alpha=1$. We again find that compared to $2 \mathrm{D}$, the LLPH method fares better in three dimensions and the accuracy becomes even better as $R$ increases. Again we may conclude that for the entire range of the coupling constant, the LLPH results can be considered to be reasonably trustworthy. The reason we are vouching for the LLPH method though it is not all that accurate is that it has certain advantages. As we have already pointed out, being a Ritz variational method, in this method we have to prescribe the wave function and therefore we can obtain several other polaronic properties using this method 


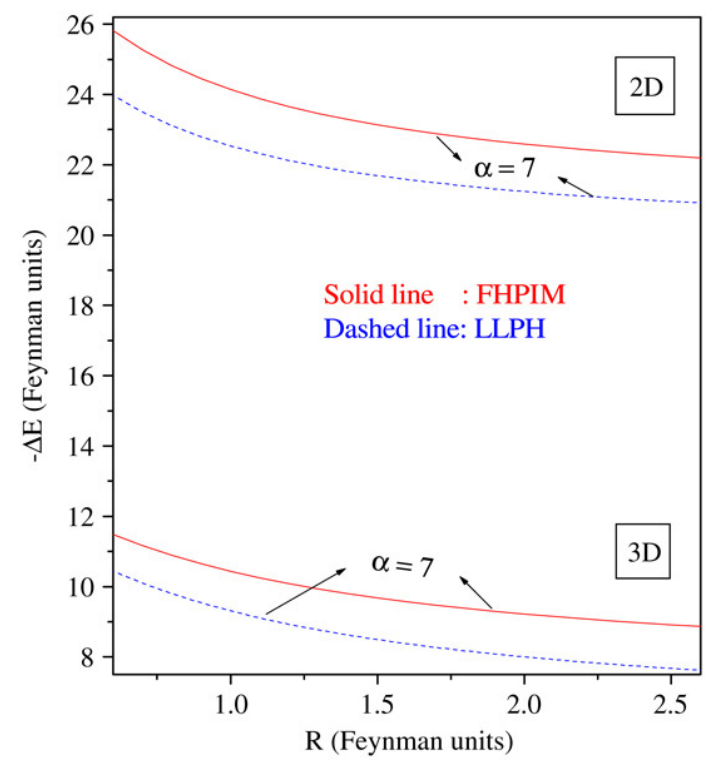

Fig. 5. Polaron self-energy, $-\Delta E$ in Feynman units as a function of $R$ for $\alpha=7$ and $V_{0}=10$ both for 2D and 3D dots obtained from FHPIM and the LLPH method.

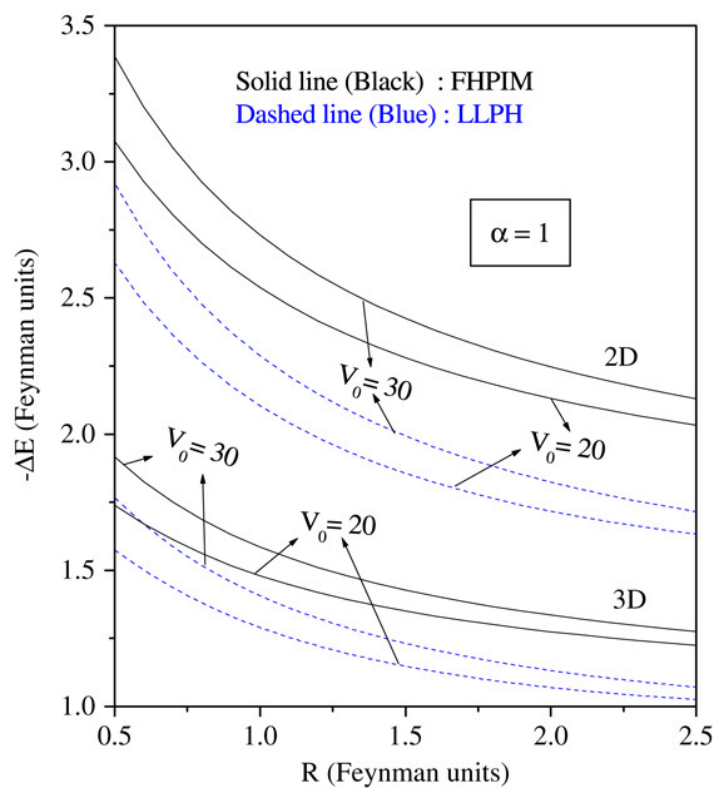

Fig. 6. Polaron self-energy, $-\Delta E$ in Feynman units as a function of $R$ for $\alpha=1$ and for three values of $V_{0}$ obtained from LLPH, and FHPIM for both 2D and 3D quantum dots.

very easily, namely the quantities that require averaging with respect to the wave function of the system.

In Section 3.3, we have already obtained the formal expressions for the number of phonons in the polaron, the radius of the polaron and the polarization potential within the framework 

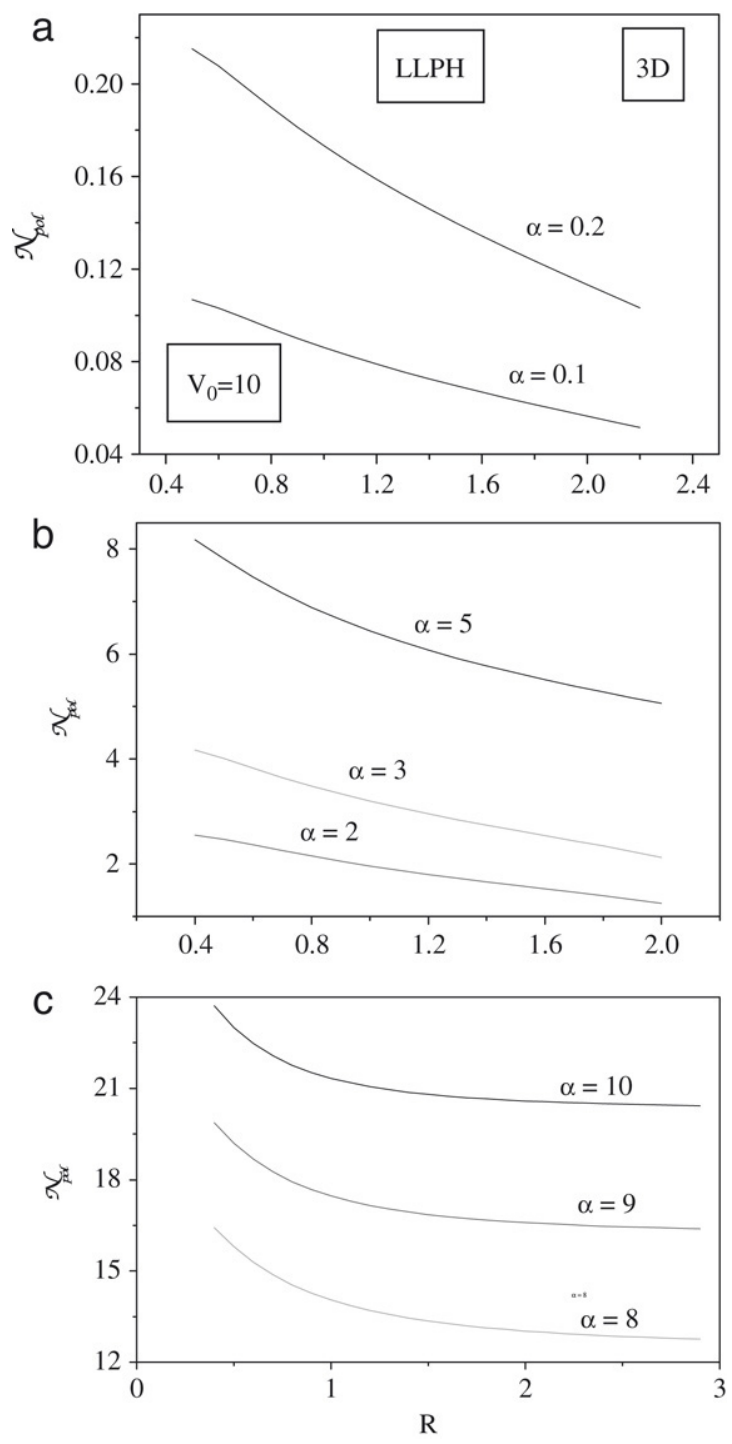

Fig. 7. Variation of the number of phonons in the polaron as a function of $R$ for different values of $\alpha$ and for $V_{0}=10$ in $3 \mathrm{D}$. (a) refers to the weak-coupling regime, (b) describes the intermediate-coupling range, and (c) shows the behaviour in the strong-coupling region.

of the LLPH method. We have to determine their numerical values using the optimized values of the variational parameters $\delta$ and $t$. Now we shall turn to discuss these numerical results.

Fig. 7 shows the behaviour of the number of phonons, $N_{\text {pol }}$ as a function of $R$. In Fig. 7(a) we show the variation of $N_{\text {pol }}$ in the weak-coupling range and in 3D. In Fig. 7(b) we consider the intermediate-coupling range, while the strong-coupling case is displayed in Fig. 7(c). In all these figures we observe that the number of phonons increases with decreasing $R$. This implies that for the same value of $V_{0}$, as the range of the confining potential $R$ decreases, the polaronic state 


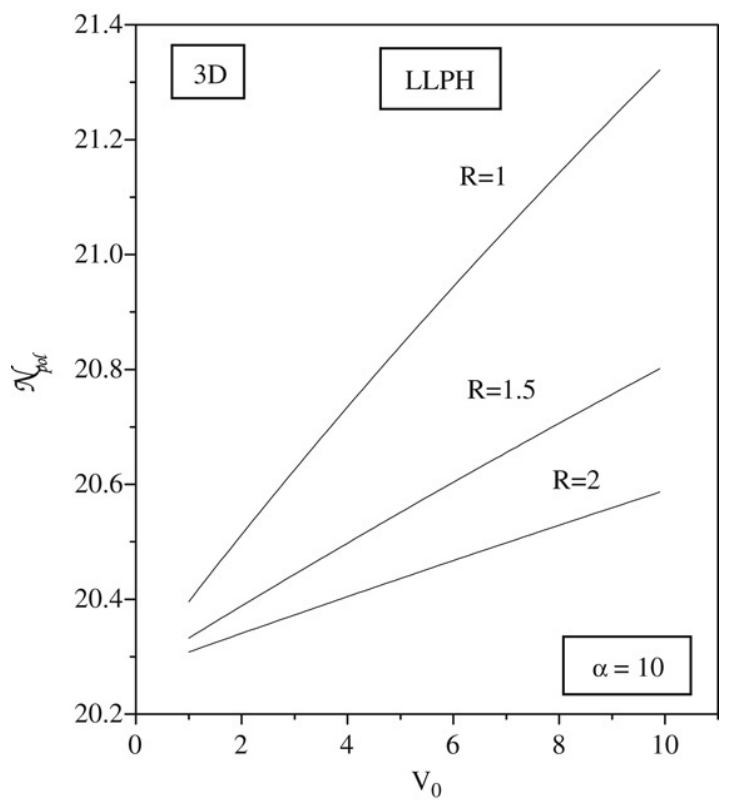

Fig. 8. Variation of the number of phonons as a function of $V_{0}$ for different values of $R$ in 3D.

becomes more and more bound with a large number of virtual phonons in the polaron cloud. This is a very interesting situation, because in this case the fluctuation in the number of phonons in the polaron is small, but still it corresponds to a highly quantum domain in terms of the length scale. We further observe that for very low values of $\alpha$, the behaviour is approximately linear. However, as $\alpha$ increases, the increase in the number of phonons with decreasing $R$ becomes more rapid. This is clearly visible in Fig. 7(c). This behaviour is of course consistent with the self-energy behaviour. We have not shown the behaviour in $2 \mathrm{D}$ for the paucity of space, but the qualitative behaviour is the same, the difference being that the effects are stronger in 2D. From now on we consider only the 3D case to save space. In Fig. 8 we display the variation of the number of phonons as a function $V_{0}$ for three values of $\mathrm{R}$. The number of phonons is found to increase with increasing $V_{0}$ and decrease with increasing $R$. The variation of $N_{\text {pol }}$ is essentially linear in $V_{0}$. As the potential, $V_{0}$ becomes deeper and deeper, the polaron state forms at lower and lower energy leading to a self-trapped state. In Fig. 9 we plot the number of phonons, $N_{\text {pol }}$ as a function of the electron-phonon coupling constant, $\alpha$ for a few values of $R$. Again, $N_{\text {pol }}$ is found to be an increasing function of $\alpha$. For small values of $\alpha$, we find that the curves look more or less linear which is of course an expected behaviour at least for small $V_{0}$. For large $V_{0}$, however, $N_{\text {pol }}$ becomes a rapidly increasing function of $\alpha$, because the polarization potential which is a function of $\alpha$ and the confining potential act in this case synergistically. For large values of $\alpha$, $N_{\text {pol }}$ of course increases very rapidly even for reasonable values of $V_{0}$ and $R$. In Fig. 10 we study the nature of the polaron size, $R_{\text {pol }}$ as a function of the range $R$ in three dimensions. We study the behaviour for the weak, intermediate and strong-coupling regimes. Fig. 10(a) shows the behaviour for $\alpha=0.01$ and 0.02 . The polaron radius increases with increasing $R$ and $\alpha$ dependence is almost negligible in the weak-coupling limit. In Fig. 10(b) we investigate the nature of the variation for the intermediate-coupling region, namely for $\alpha=2,3$ and 5. Here we find an interesting behaviour. For small $R$, the behaviour is almost linear, but as $R$ increases, for 


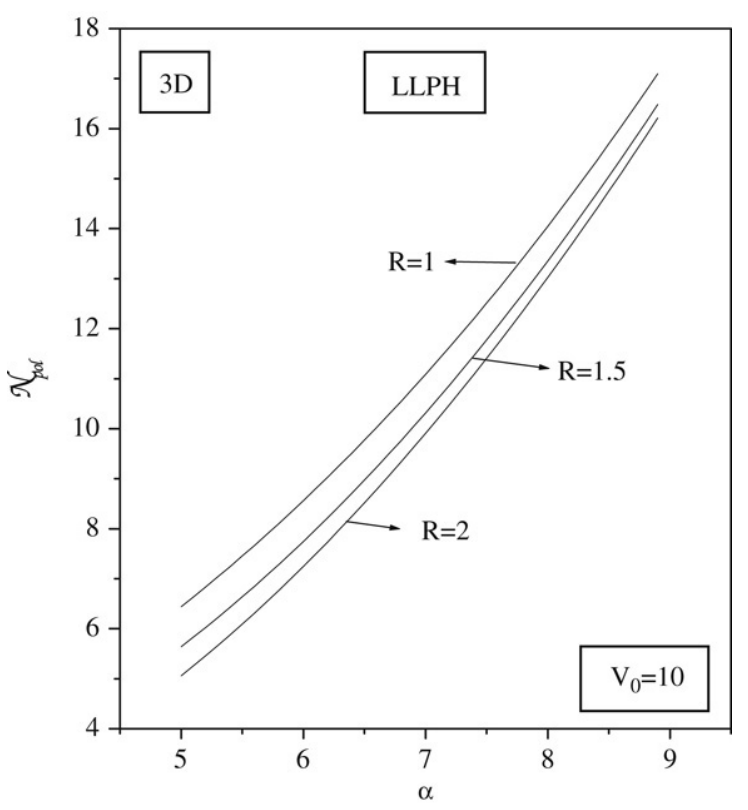

Fig. 9. Variation of the number of phonons as a function of $\alpha$ for different values of $R$.

$\alpha=2$ and 3 , the increase in the polaron size becomes a little more rapid than the linearity, while for $\alpha=5$, it appears that the polaron radius tends to saturate to some smaller value. This may be indicative of the onset of the strong-confinement regime. Thus $\alpha=5$ appears to belong to the strong-coupling region in the present situation because of the seemingly qualitative difference in the polaronic behaviour in this case. In Fig. 10(c) we plot the polaron radius for strong-coupling values namely, $\alpha=8,9,10$. It is now quite clear that the polaron size saturates with $R$. The limiting polaron size is of course smaller for larger values of $\alpha$. In Fig. 11 we study the behaviour of the effective polaron radius, $R_{\mathrm{pol}}$ as a function of the depth of the confining potential, $V_{0}$ for a few values of $R$. As the depth, $V_{0}$ increases, the polaron size becomes smaller and smaller monotonically. The decrease is almost linear. However, for smaller $R$ values, the decrease is much faster, albeit linear. This is of course understandable, because as the confining potential becomes deeper and deeper with the range $R$ remaining the same, the polaron sinks more and more towards the bottom of the potential producing a GS at a lower energy and consequently the size of the polaron decreases. With a lower value of $R$, there would be a combined effect of $R$ and $V_{0}$ leading to a rapid fall in the polaron size. This is the strong-confinement regime.

Fig. 12 shows the dependence of the polaron size, $R_{\text {pol }}$ on $\alpha$ for a few values of $R$. One can observe that at small $\alpha$, the polaron size is relatively larger, but it decreases monotonically as $\alpha$ increases, apparently saturating to some common value independent of $R$. This behaviour may be explained in the following way. At large $\alpha$, the polaron goes into a self-trapped localized state and consequently, its size becomes very small and in the limiting case, $R_{\text {pol }}$ saturates to around one lattice spacing. In this limit, the range of the potential becomes irrelevant and the polaron size becomes independent of $R$. This is the strong-coupling limit. This is in principle different from the strong-confinement case discussed above, although in both cases the polaron is described by a strongly localized state. Nevertheless, apparently, both large 

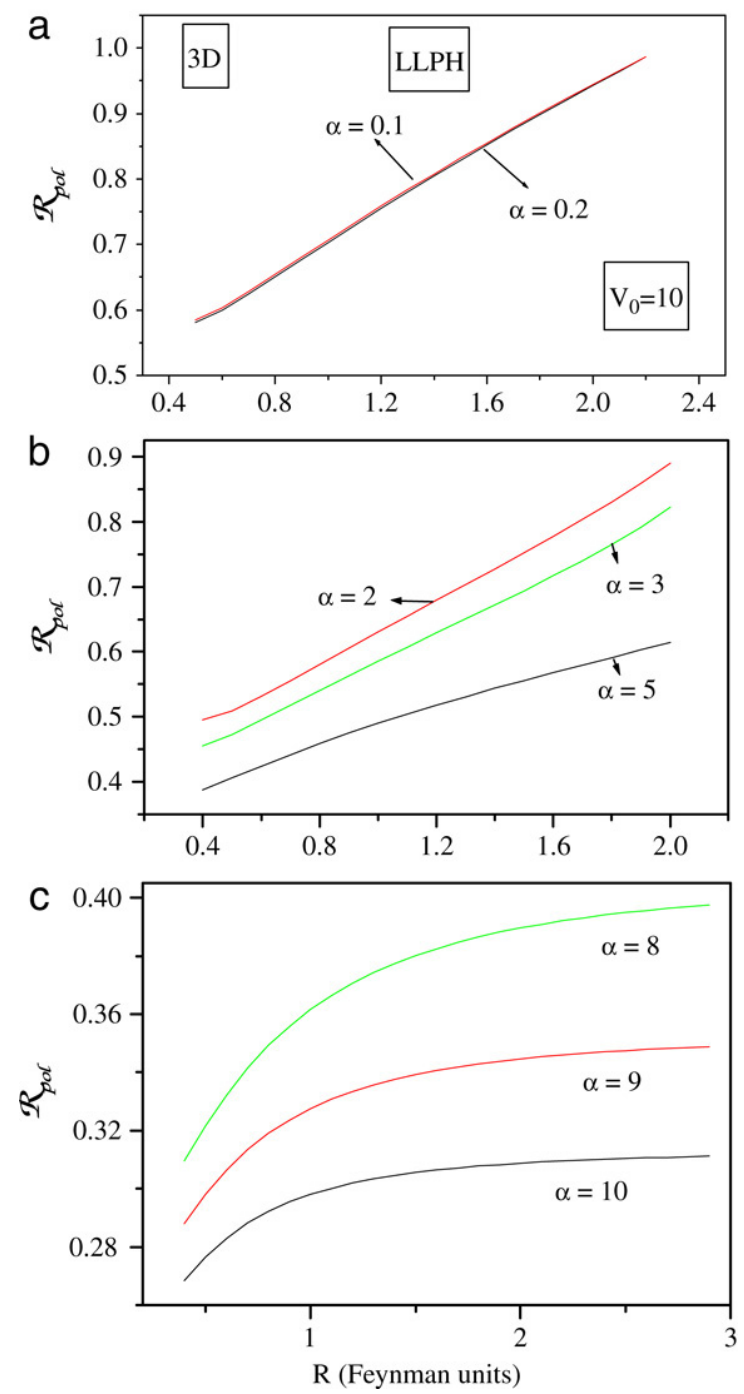

Fig. 10. Variation of the polaron size in 3D. a, b, c show the behaviour as a function of $R$ for different values of $\alpha$.

$\alpha$ and small $R$ essentially produce a quantitatively similar behaviour, because both lead to localization.

We next study the behaviour of the polarization potential, $-e V(r)$ as a function of $r$. In Fig. 13 we show the variation of the polarization potential as a function of $r$ for a few values of the depth of the potential $V_{0}$. We observe that as the confining potential becomes deeper and deeper, the polarization potential also deepens, more so at smaller $r$, to accommodate more and more bound states. In Fig. 14, we show the spatial variation of the polarization potential for a few values of $\alpha$. Again the polarization potential becomes deeper with increasing $\alpha$ to make a more favourable situation for self-trapping. Finally in Fig. 15, we study the behaviour of $-e V(r)$ for a few values of $R$. Here we find that as $R$ increases, the polarization potential becomes more and more shallow as is commonly expected. 


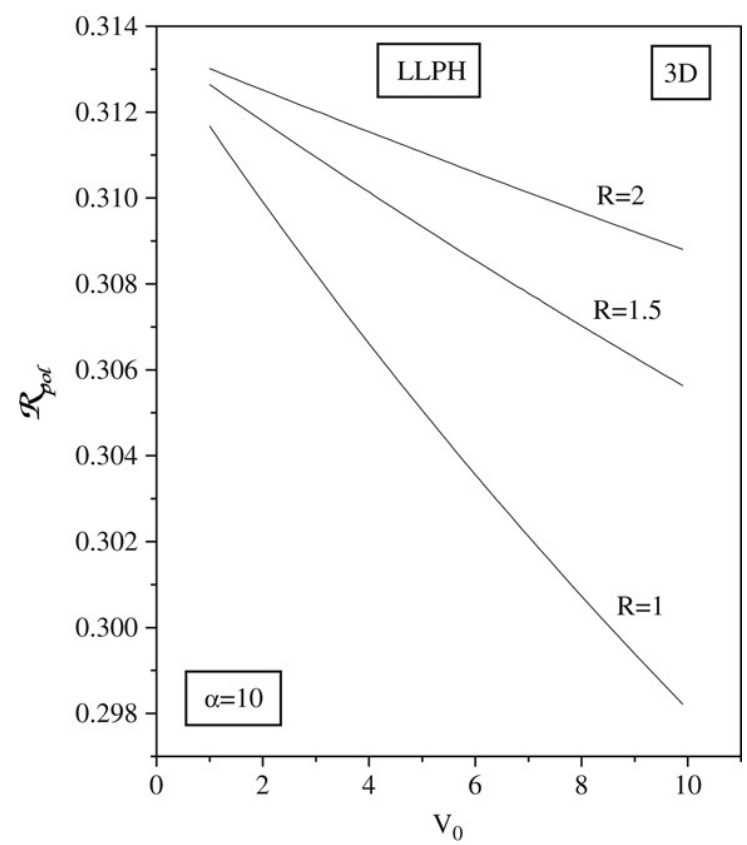

Fig. 11. Variation of the polaron size as a function of $V_{0}$ for different values of $R$.

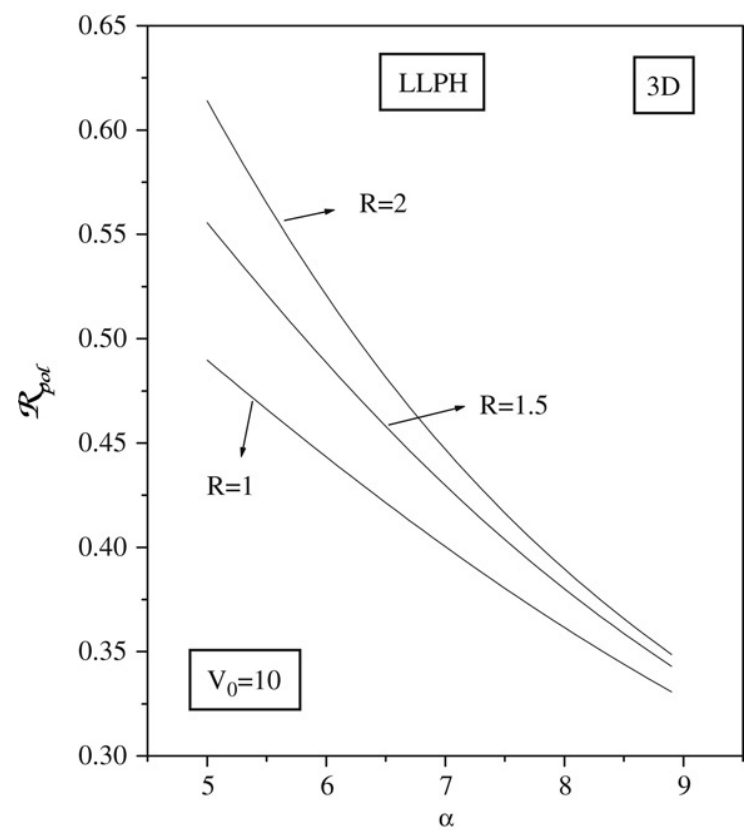

Fig. 12. Variation of the polaron size as a function of $\alpha$ for different values of $R$. The inset shows the behaviour in $2 \mathrm{D}$. 


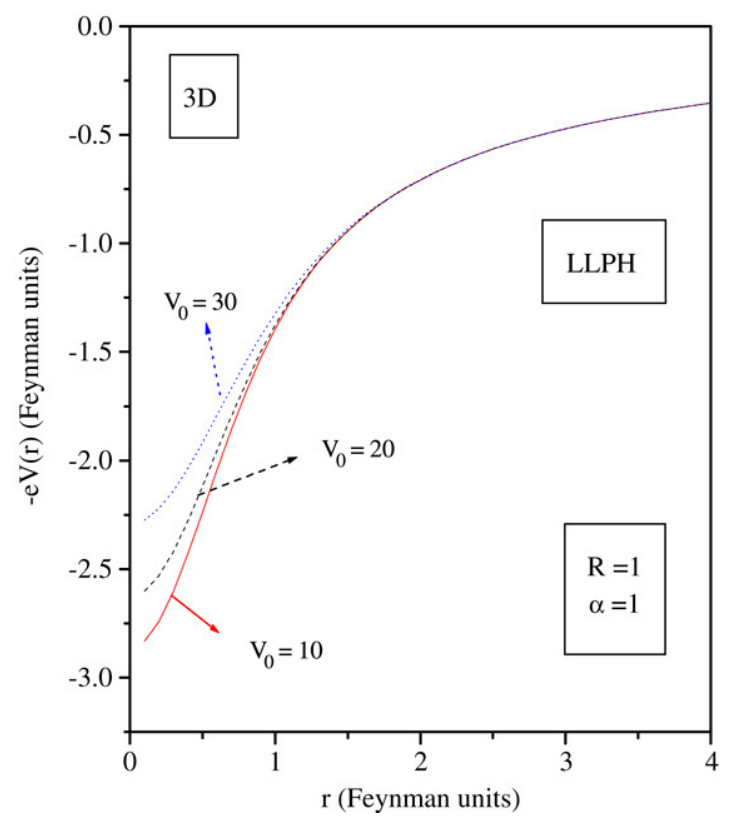

Fig. 13. Variation of the polarization potential as function of $r$ for a few values of $V_{0}$ in 3D.

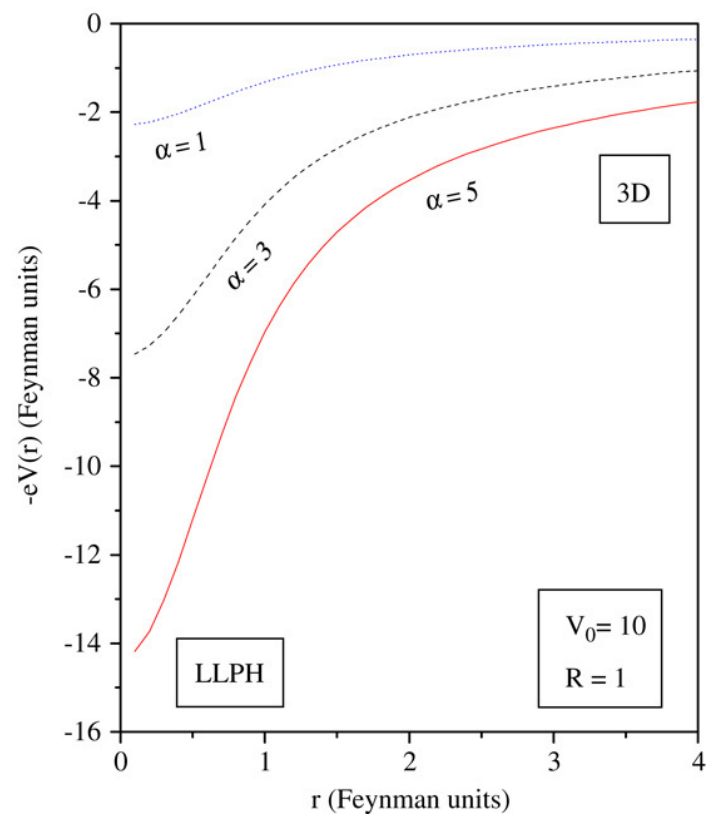

Fig. 14. Variation of the polarization potential as function of $r$ for a few values of $\alpha$ in 3D.

\section{Conclusion}

We have studied the problem of an electron interacting with the LO phonons in a quantum dot with a symmetric confinement by a Gaussian potential. We have considered both 2D and 


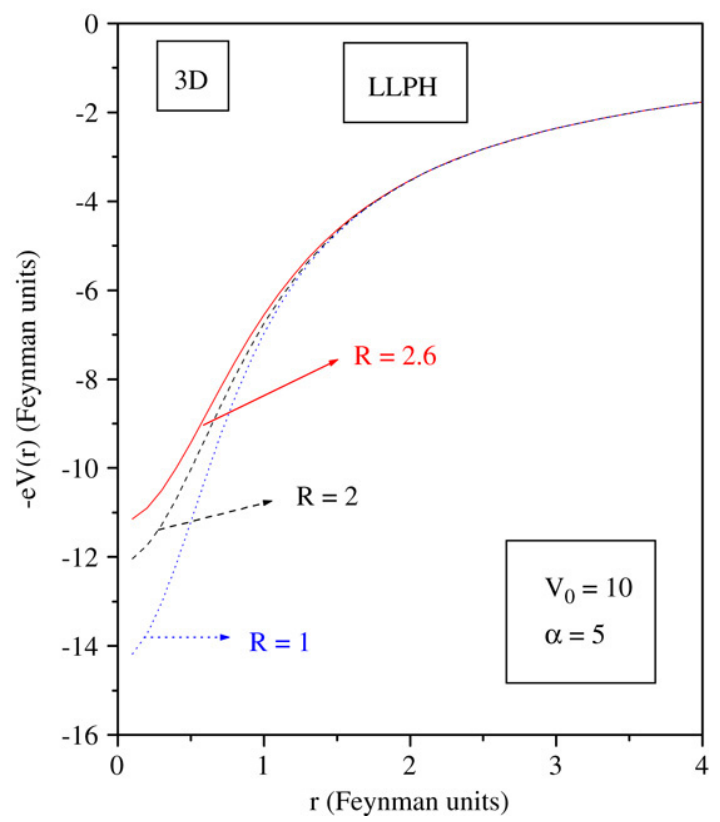

Fig. 15. Variation of the polarization potential as function of $r$ for a few values of $R$ in 3D.

3D systems and applied the perturbation theory, the LLPH method and the Feynman-Haken path-integral technique to find out the ground state energy of the system and hence the polaron self-energy for the entire range of the electron-phonon coupling and for arbitrary confinement. Since the Gaussian potential is not exactly soluble, we performed an approximate second-order perturbative calculation using a mean-field kind of treatment. We have shown that the Gaussian confining potential provides a stronger polaronic binding for a larger range of the potential as compared to the parabolic potential, while for smaller values of $\mathrm{R}$ the parabolic potential gives a much stronger binding. The qualitative behaviour is same in both $2 \mathrm{D}$ and $3 \mathrm{D}$ dots, but the discrepancy between the results for the Gaussian potential and the parabolic potential is more in two dimensions than in three dimensions. Thus we have shown that the parabolic potential is a poor approximation of the more realistic Gaussian potential for the entire range of $\mathrm{R}$. We have shown that the polaronic effects become stronger as $V_{0}$ increases and again the effect is much stronger in $2 \mathrm{D}$ than in $3 \mathrm{D}$. We have compared the polaron self-energy results obtained from the perturbation theory, FHPIM and LLPH methods. In the weak-coupling regime, RSPT results give the strongest polaronic binding and the LLPH method suggests the weakest binding. The results from FHPIM lie somewhere in the middle, or more precisely, a little closer to the RSPT results than to the LLPH curves. We have ascribed the success of RSPT to our meanfield approximation. The 'not so great success' of the LLPH method may be attributed to the poor choice of the trial electronic function which does not include the effect of anharmonicity present in the Gaussian confinement. However all the methods show qualitatively the same behaviour, namely that the polaronic effects increase with decreasing $\mathrm{R}$, increase with increasing $\alpha$ and are larger in 2D than in 3D. In the intermediate- and strong-coupling regimes, again the FHPIM provides better results than the LLPH method for the same reason as mentioned above. Because of the simplicity of the LLPH method, we have used this method to find out the number of phonons in the cloud around the polaron, the polaron size and the polarization potential. 
We have shown that the number of phonons increases with increasing $\alpha$, decreases with increasing $R$, and increases with increasing $V_{0}$. In the weak-coupling region, the decrease in $N_{\text {pol }}$ is almost linear in $\alpha$, but in the strong-coupling range, $N_{\text {pol }}$ tends to saturate to a constant depending on the value of $\alpha$. The behaviour of $N_{\text {pol }}$ with $V_{0}$ is also almost linear, while as a

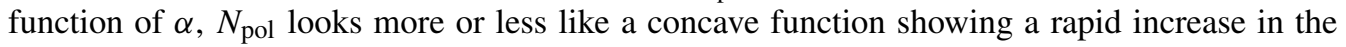
number of phonons at large $\alpha$. The polaron size also shows some interesting behaviour. In the weak-coupling region it increases linearly with $R$, but seems to be essentially independent of $\alpha$. This is reminiscent of the delocalized large polaron scenario. In the intermediate-coupling range, $R_{\text {pol }}$ shows a small bending upwards at large $R$, while for strong coupling, the polaron size above some value of $R$ tends to saturate to some constants which depend on the value of $\alpha$. As a function of $V_{0}$, we have shown that, $R_{\text {pol }}$ decreases almost linearly leading to localization, the decrease being more rapid for smaller $R$ values. $R_{\text {pol }}$ also decreases with increasing $\alpha$, tending to saturate to some constant value independent of $R$. On the other hand, below some value of $\alpha$, the polaron size increases very rapidly with decreasing $\alpha$, more so for larger $R$, again leading to the delocalized large polaron. Of course the polaron size is always limited by the quantum dot size or in other words by the range of the confining potential. We have determined the shape of the polarization potential using the LLPH method. There is clear indication of a minimum structure at the centre of the dot. The minimum becomes deeper and deeper with increasing $V_{0}$ and $\alpha$ and decreasing $R$. At large $r$, however, the polarization potential does not depend on the range or depth of the potential. All these behaviour are also expected to show up in a 2D quantum dot, but of course with a stronger effect. Finally, we should mention that for a very small dot, tunnelling becomes important and in that case, interaction with interface phonons will play an important role. We have however neglected this interaction for the sake of simplicity but we believe that the essential qualitative features of the problem will remain more or less the same even after the inclusion of the interface phonons.

\section{References}

[1] M.A. Kastner, Rev. Modern Phys. 64 (1992) 849;

N.F. Johnson, J. Phys.: Condens. Matter 7 (1995) 965;

R.C. Ashoori, Nature 379 (1996) 413;

U. Woggon, Optical Properties of Semiconductor Quantum Dots, Springer, Berlin, 1997;

L. Jack, P. Hawrylack, A. Wojs, Quantum dots, Springer, Berlin, 1998;

D. Bimberg, M. Grundmann, N.N. Ledentsov, Quan dot Heterostructures, Wiley, New York, 1998;

S. Mukhopadhyay, A. Chatterjee, Acta Phys. Pol. B 32 (2001) 473.

[2] Ch. Sikorski, U. Merkt, Phys. Rev. Lett. 62 (1989) 2164; Surf. Sci. 229 (1990) 282.

[3] B. Meurer, D. Heitmann, K. Ploog, Phys. Rev. Lett. 68 (1992) 1371.

[4] R.C. Ashoori, H.L. Stormer, J.S. Weiner, L.N. Pfeiffer, S.J. Pearton, K.W. Balswin, K.W. West, Phys. Rev. Lett. 68 (1992) 3088.

[5] H. Drexler, D. Leonard, W. Hansen, J.P. Kotthaus, P.M. Petroff, Phys. Rev. Lett. 73 (1994) 2252.

[6] W. Kohn, Phys. Rev. 123 (1961) 1242.

[7] F.M. Peeters, Phys. Rev. B 42 (1990) 1486.

[8] S.K. Yip, Phys. Rev. B 43 (1991) 1707.

[9] Q.P. Li, K. Karrai, S.K. Yip, S. Das Sarma, H.D. Drew, Phys. Rev. B 43 (1991) 5151.

[10] P.A. Maxym, T. Chakraborty, Phys. Rev. Lett. 65 (1990) 108;

S.W. Gu, K.X. Guo, Solid State Commun. 89 (1993) 1023;

N.F. Johnson, M.c. Payne, Phys. Rev. Lett. 67 (1991) 1157;

N.F. Johnson, F.C. Payne, Phys. Rev. B 45 (1992) 3819;

R. Egger, W. Hausler, C.H. Mak, H. Grabert, Phys. Rev. Lett. 82 (1999) 3320;

K.D. Zhu, S.W. Gu, Phys. Rev. B 47 (1993) 12947;

K.D. Zhu, T. Kobayashi, Solid State Commun. 95 (1995) 805; 
C.Y. Chen, P.W. Jin, W.S. Li, D.L. Lin, Phys. Rev. 56 (1997) 14913;

S. Mukhopadhyay, A. Chatterjee, Phys. Lett. A 242 (1998) 355; Phys. Lett. A 204 (1995) 411; Internat. J. Modern Phys. 10 (1996) 2781; Phys. Rev. B 55 (1997) 9297; Phys. Rev. B 58 (1998) 2088; Phys. Lett. A 240 (1998) 100; Phys. Rev. B 59 (1999) R7833; J. Phys.: Condens. Matter 11 (1999) 2071; Internat. J. Modern Phys. B 14 (2000) 3897; Internat. J. Modern Phys. B 16 (2002) 1489;

N. Kervan, T. Altanhan, A. Chatterjee, Phys. Lett. A 315 (2003) 280;

P.M. Krishna, A. Chatterjee, Physica B 358 (2005) 191; Physica E 30 (2005) 64;

P.M. Krishna, S. Mukhopadhyay, A. Chatterjee, Solid State Commun. 138 (2006) 285;

Phys. Lett. A 360 (2007) 655.

[11] D. Heitmann, K.K. Bollweg, V. Gudmundsson, T. Kurth, S.P. Riege, Physica E 1 (1997) 204;

B.T. Miller, W. Hansen, S. Manus, R.J. Luyken, A. Lorke, J.P. Kotthaus, S. Huant, G. Mediros-Ribeiro, P.M. Petroff, Phys. Rev. B 56 (1997) 6764.

[12] B. Szfran, J. Adamowsky, S. Bednarek, Physica E 4 (1999) 1.

[13] S. Bednarek, B. Szafran, J. Adamowsky, Phys. Rev. B 59 (1999) 13036.

[14] B. Szfran, J. Adamowsky, S. Bednarek, Phys. Rev. B 61 (2000) 1971.

[15] J. Adamowsky, M. Sobkowicz, B. Szafran, S. Bednarek, Phys. Rev. B 62 (2000) 4234.

[16] N. Bessis, H. Friedrich, C. Wheatly, J. Phys. A 15 (1982) 3679;

C.S. Lai, J. Phys. A 16 (1983) L181;

R.E. Crandall, J. Phys. A 16 (1983) L395;

M. Cohen, J. Phys. A 17 (1984) L101;

A. Chatterjee, J. Phys. A 18 (1985) 2403; Phys. Rep. 186 (1990) 249.

[17] T.K. Mitra, A. Chatterjee, S. Mukhopadhyay, Phys. Rep. 153 (1987) 91.

[18] H. Fröhlich, Phil. Mag. Suppl. 3 (1954) 325.

[19] R.P. Feynman, Phys. Rev. 97 (1955) 660.

[20] F.M. Peeters, Wu Xiaoguang, J.T. Devreese, Phys. Rev. B 33 (1986) 3926.

[21] M. Abramowich, A. Stegun (Eds.), Handbook of Mathematical Functions, Dover, New York, 1965, p. 257.

[22] Z. Haken, Z. Phys. 147 (1957) 323.

[23] T.D. Lee, F. Low, D. Pines, Phys. Rev. 90 (1953) 297.

[24] W. Huybrechts, J. Phys. C: Solid State Phys. 10 (1977) 3761. 